\title{
On the Variations in Mutual Information of MIMO Communication Systems Due to Perturbed Channel State Information at Transmitter
}

\author{
M. R. Bhavani Shankar and K. V. S. Hari, Senior Member, IEEE
}

\begin{abstract}
In this paper, we address the issue of perturbed information at the transmitter in a multiple-input multiple-output (MIMO) system. Two schemes, correlation matrix feedback and channel information feedback, are proposed to convey information necessary for achieving capacity to the transmitter. This information is perturbed due to various impairments. A perturbation analysis is carried out to study the variations in mutual information for each of the proposed schemes. For ergodic channels, this analysis is used to design a MIMO system with a limited rate feedback. Using a codebook-based approach, vector quantizers are designed to minimize the loss in ergodic capacity for each of the proposed schemes, and their performance is studied. A non-codebook method, parameter-based feedback, is also presented, and the underlying rate-performance tradeoff is highlighted.
\end{abstract}

Index Terms-Channel state information (CSI), ergodic capacity, limited-rate feedback, multiple-input multiple-output (MIMO) system, perturbation analysis, perturbed information, vector quantization.

\section{INTRODUCTION}

$\mathbf{E}$ XPRESSIONS for mutual information of high-rate multiple-input multiple-output (MIMO) systems with perfect channel state information (CSI) at receiver (CSIR) and no CSI at the transmitter (CSIT) have been presented in [1] and [2]. The use of perfect CSIT along with perfect CSIR has been shown to improve the achievable data rates [3] of such systems. However, in practice, CSIT and/or CSIR is not perfect, and expressions for capacity of MIMO Gaussian flat-fading channels with imperfect CSIT and/or CSIR have been summarized in [4]. In particular, the channel capacity with partial CSIT (in the form of channel mean and covariance information) and perfect CSIR is considered in [4] and [5]. Bounds on mutual information with imperfect CSIR and no CSIT are derived in [6]. The case of identical, imperfect channel estimates at the transmitter and receiver was considered to derive bounds on the achievable capacity/outage probability in [7]. The key assumption in [7] is that CSIT and CSIR are identical. However, in practice, this assumption is not true, due to impairments like rate constraint and delay on the feedback link. Further, these impairments present imperfect (perturbed) information to the transmitter.

Paper approved by R. Raheli, the Editor for Detection, Equalization, and Coding of the IEEE Communications Society. Manuscript received November 28, 2002; revised March 5, 2004; August 29, 2005; and February 22, 2006. This paper was presented in part at the IEEE International Conference on Personal Wireless Communication, New Delhi, India, December 2002

The authors are with the Department of Electrical Communication Engineering, Indian Institute of Science, Bangalore 560 012, India (e-mail: bshankar@ece.iisc.ernet.in; hari@ece.iisc.ernet.in).

Digital Object Identifier 10.1109/TCOMM.2006.881240
References [8]-[14] study the performance of multiple-antenna systems with perturbed CSIT for specific forms of feedback over limited-rate links. Feedback of beamforming vector for multiple-input single-output (MISO) systems is considered in [8]-[10]. Feedback of eigenvectors of the input correlation matrix followed by equal power allocation on useful modes for MIMO systems is considered in [11]. The random vector quantization (RVQ) scheme, which uses feedback bits to specify the columns of the input correlation matrix from a set of random vectors, is proposed and an asymptotic analysis of mutual information is undertaken in [12]. This scheme is appropriate for systems with a large number of antennas. In [13], feedback of channel gain for MISO systems is considered. Feedback of input correlation matrices satisfying an average power constraint over a coding block is considered in [14] as a capacity-achieving strategy. Inherently linked to this study is a methodology for the design of multiple-antenna systems with limited-rate feedback. The issues involved in the design of such systems and the use of intelligent vector quantizers (VQs) [15] for feedback are highlighted in [16]. Two approaches are pursued in the literature for the design of such VQs. One approach is to obtain expressions for loss in ergodic capacity due to quantized CSIT, and then design a VQ which minimizes this loss [10], [11]. Another approach is to optimize the VQ parameters to maximize the performance metric [8], [9], [14]. The parameters of VQ are optimized to enhance the received signal-to-noise ratio (SNR) in [8], and to reduce outage probability in [9]. The problem of designing a VQ for the feedback of input correlation matrix with an aim of maximizing the mutual information is posed in [8] and [14]. However, this problem does not yield efficient VQs due to the inability to obtain a closed-form expression for the centroid while employing the Lloyd-Max iterative algorithm [15]. As a result, the problem was not solved in [8], while a heuristic approximation to the cost function was used to compute the centroid in [14]. This heuristic approximation results in a lower bound on forward channel capacity [14].

In this paper, we consider two feedback schemes that convey complete instantaneous information needed to achieve the MIMO ergodic capacity. The effect of perturbed CSIT (possibly due to limited-rate feedback) on mutual information is analyzed for these schemes. Expressions and bounds on mutual information are derived, assuming a fixed (deterministic) channel with unknown perturbations. Following the approach of minimizing the loss in ergodic capacity caused by quantized CSIT, we use the derived results to obtain efficient VQ designs for feedback over limited-rate links.

The main contributions of the paper are summarized below. 


\section{A. Proposed Feedback Schemes}

The first scheme, correlation matrix feedback, involves the feedback of the input correlation matrix satisfying an average power constraint per transmission. This scheme is different from the channel covariance feedback discussed in [4], which involves the channel covariance matrix. The second scheme, channel information feedback, involves feedback of information about the instantaneous channel realization necessary for signal shaping. Unlike [11] and [12], these schemes convey complete information to the transmitter.

\section{B. Design of VQ for Proposed Feedback Schemes}

For each of the proposed schemes, VQs are designed for feedback over limited-rate links. Since the heuristic approximation in [14] is used only for centroid computation and not for the entire VQ design, monotonic convergence of the iterative algorithm is not guaranteed. Unlike [8] and [14], the VQs designed in this paper have a closed-form expression for the centroid and preserve the monotonicity of Lloyd-Max iterations. The performance of the proposed quantizers are numerically compared with that of existing ones, and the gains in mutual information with feedback are highlighted.

\section{Parameter-Based Feedback}

Motivated by parameterization presented in [17], we also present parameter-based feedback, a non-codebook scheme that can be used to obtain a tradeoff between the number of parameters fed back and the loss in capacity.

Notations: Matrices are indicated by uppercase boldface letters. $\lambda_{i}(\mathbf{Y})$ and $\mathbf{Y}(i, j)$ denote, respectively, the $i$ th eigenvalue and $(i, j)$ th entry of a matrix $\mathbf{Y} . \mathbf{Y}^{\dagger}$ refers to the pseudoinverse of $\mathbf{Y} . \Lambda_{Y}$ is the eigenvalue matrix of $\mathbf{Y}$ with $\left\{\lambda_{i}(\mathbf{Y})\right\}$ as diagonal entries. Further, $\|\mathbf{Y}\|$ denotes the spectral norm [18], and $\|\mathbf{Y}\|_{F}$ denotes the Frobenius norm [18] of $\mathbf{Y}$. For any positive (semi) definite matrix $\mathbf{Y},\left\{\lambda_{i}(\mathbf{Y})\right\}$ 's are arranged in descending order. $\mathbf{I}_{n}$ is an $n \times n$ identity matrix. $\mathbf{Y} \geq \mathbf{0}(\mathbf{Y}>\mathbf{0})$ means that $\mathbf{Y}$ is positive semidefinite (positive definite). $\mathbf{Y}=\mathbf{V} \boldsymbol{\Lambda}_{Y} \mathbf{V}^{*}$, $\mathbf{Y}^{1 / 2}=\mathbf{V} \Lambda_{Y}^{1 / 2} \mathbf{V}^{*}$, where $\Lambda_{Y}^{1 / 2}(i, i)=\sqrt{\Lambda_{Y}(i, i)}$. det denotes determinant, Trace denotes the trace, $E[\cdot]$ denotes the expectation operator, and ln denotes natural logarithm. Further, $i \in[1, p]$ implies $i=1,2, \ldots, p, \operatorname{diag}(a, b, \ldots, c)$ refers to a diagonal matrix with entries $a, b, \ldots, c$, w.r.t. stands for "with respect to," "s.t." means "subject to," "i.i.d." refers to independent and identically distributed, and SISO refers to single-input single-output.

\section{SYSTEM MODEL}

We consider the MIMO system with $m$ transmit and $n$ receive antennas over a Gaussian channel. Let $\mathbf{H}$ denote the $n \times m$ channel matrix with complex coefficients, $\underline{x}$ be the zero-mean input vector with correlation matrix $\mathbf{Q}$, and $y$ be the received vector. The system model is

$$
\underline{y}=\mathbf{H} \underline{x}+\underline{\eta}
$$

where $\eta$ is the receiver noise modeled as a zero-mean complex circular Gaussian random vector with correlation matrix $\mathbf{I}_{n}$.

The channel is assumed to be flat and deterministic (fixed) initially. Extensions to ergodic channels are subsequently provided. The power available for transmission is $P$, so that Trace $(\mathbf{Q})=P$. The mutual information for such a set up is $\log \operatorname{det}\left[\mathbf{I}_{n}+\mathbf{H Q H}^{*}\right]$ [1], where $\log _{2}$ is written as $\log$ and ${ }^{*}$ denotes the Hermitian operator. Denote $\mathbf{A}=\mathbf{H}^{*} \mathbf{H}$. Let $\mathbf{A}=\mathbf{V} \boldsymbol{\Lambda}_{A} \mathbf{V}^{*}$ denote the eigenvalue decomposition (EVD) [18] of $\mathbf{A}$, and denote $\mathbf{A}^{1 / 2}=\mathbf{V} \Lambda_{A}^{1 / 2} \mathbf{V}^{*}$. With perfect CSIT, the capacity-achieving matrix $\mathbf{Q}_{\mathrm{opt}}$ has the EVD $\mathbf{Q}_{\mathrm{opt}}=\mathbf{V} \boldsymbol{\Lambda}_{Q} \mathbf{V}^{*}$, where $\boldsymbol{\Lambda}_{Q}$ is obtained by waterfilling [1]. The capacity for the above setup is

$$
C_{\mathrm{opt}}=\log \operatorname{det}\left[\mathbf{I}_{n}+\mathbf{H} \mathbf{Q}_{\mathrm{opt}} \mathbf{H}^{*}\right]=\log \operatorname{det}\left[\mathbf{I}_{m}+\mathbf{A}^{1 / 2} \mathbf{Q}_{\mathrm{opt}} \mathbf{A}^{1 / 2}\right]
$$

From the form of $\mathbf{Q}_{\mathrm{opt}}$, it is clear that the transmitter must have perfect information of $\mathbf{H}$ (or $\mathbf{A}$ ) to achieve $C_{\mathrm{opt}}$. Generally, this information is obtained from the receiver through feedback. However, due to feedback rate constraint, delay, and other impairments, the transmitter is presented with perturbed information; for example, $\mathbf{A}+\boldsymbol{\Delta} \mathbf{A}$ instead of $\mathbf{A}$. Assuming the available information to be true, the transmitter derives perturbed correlation matrix $\widehat{\mathbf{Q}}_{\mathrm{opt}}$ from perturbed information for signal shaping. The resulting mutual information is $C$, given below as

$$
C=\log \operatorname{det}\left[\mathbf{I}_{m}+\mathbf{A}^{1 / 2} \widehat{\mathbf{Q}}_{\mathrm{opt}} \mathbf{A}^{1 / 2}\right]
$$

Clearly, $C \leq C_{\mathrm{opt}}$, thereby resulting in a loss. We propose two simple feedback schemes: 1) the correlation matrix feedback scheme where the correlation matrix $\mathbf{Q}$ is fed back; and 2) the channel information feedback scheme where information about the channel is fed back. We analyze the effect of perturbed information at the transmitter for each of the proposed schemes to understand the extent of loss in mutual information. This analysis is presented in Section III. The analysis is then used in Section IV to design a system with feedback to minimize the loss in ergodic capacity.

\section{PERTURBATION ANALYSIS}

An analysis for arbitrary perturbations is, in general, difficult. Since small perturbations are amenable to certain approximations which result in a tractable analysis, most of the analysis in this section deals with small perturbations. We begin with the feedback of the correlation matrix.

\section{A. Correlation Matrix Feedback}

Let $\widehat{\mathbf{Q}}_{\mathrm{opt}}$ be the perturbed correlation matrix available at the transmitter due to feedback, while $\mathbf{Q}_{\mathrm{opt}}$ is the optimal correlation matrix for $\mathbf{H}$. The perturbation is $\Delta \mathbf{Q}=\widehat{\mathbf{Q}}_{\mathrm{opt}}-\mathbf{Q}_{\mathrm{opt}}$. We denote $r=\operatorname{rank}\left(\mathbf{Q}_{\mathrm{opt}}\right)$ and $s=\operatorname{rank}\left(\widehat{\mathbf{Q}}_{\mathrm{opt}}\right)$. An analysis for small perturbations is detailed. 
1) Analysis for Small Perturbations: Let $\kappa_{r}=r /(P+$ $\left.\sum_{i=1}^{r} 1 / \lambda_{i}(\mathbf{A})\right)$ and $\Sigma$ be the diagonal matrix with $\Sigma(i, i)=$ $\kappa_{r}, i \in[1, r], \boldsymbol{\Sigma}(i, i)=\lambda_{i}(\mathbf{A}), i \in[r+1, m]$. Then

$$
C=C_{\mathrm{opt}}+\log \operatorname{det}\left[\mathbf{I}_{m}+\mathbf{\Sigma} \mathbf{V}^{*}[\boldsymbol{\Delta} \mathbf{Q}] \mathbf{V}\right] .
$$

It is shown in Appendix I that $\log \operatorname{det}\left[\mathbf{I}_{m}+\Sigma \mathbf{V}^{*}[\Delta \mathbf{Q}] \mathbf{V}\right] \leq$ $\log \operatorname{det}\left[\mathbf{I}_{m}+\kappa_{r}[\boldsymbol{\Delta} \mathbf{Q}]\right]$. Then

$$
C \leq C_{\text {opt }}+\log \operatorname{det}\left[\mathbf{I}_{m}+\kappa_{r}[\boldsymbol{\Delta} \mathbf{Q}]\right]
$$

Equality occurs for $r=m$. This bound is seen to be reasonably tight from simulations. Further, the bound improves with an increase in $P$ or with a reduction in perturbation. It is also shown in Appendix I that

$$
\begin{aligned}
\operatorname{det}\left[\mathbf{I}_{m}+\kappa_{r}[\right. & \Delta \mathbf{Q}]] \\
& =\left[1-\frac{\kappa_{r}^{2}}{2}\|\Delta \mathbf{Q}\|_{F}^{2}+\frac{\kappa_{r}^{3}}{3} \operatorname{Trace}\left([\Delta \mathbf{Q}]^{3}\right)+R_{r}\right]
\end{aligned}
$$

where $R_{r}$ contains the residual terms. Hence, for small perturbations in $\mathbf{Q}_{\mathrm{opt}}$, we can approximate

$\log \operatorname{det}\left[\mathbf{I}_{m}+\kappa_{r}[\Delta \mathbf{Q}]\right] \approx \log \left[1-\frac{\kappa_{r}^{2}}{2}\|\Delta \mathbf{Q}\|_{F}^{2}\right] \approx-\frac{\kappa_{r}^{2}}{2 \ln 2}\|\Delta \mathbf{Q}\|_{F}^{2}$.

Using this approximation with (5), we have

$$
C \leq C_{\mathrm{opt}}-\frac{\kappa_{r}^{2}}{2 \ln 2}\|\Delta \mathbf{Q}\|_{F}^{2} .
$$

A lower bound on $C$ is derived in (30) of Appendix I-C. Under the small perturbation assumption, using $\log \operatorname{det}(\mathbf{I}+\mathbf{Y}) \approx$ $\log (1+\operatorname{Trace}(\mathbf{Y})) \approx \operatorname{Trace}(\mathbf{Y}),(30)$ becomes

$$
\begin{aligned}
C \geq C_{\mathrm{opt}} & -\frac{\kappa_{r}^{2}}{2 \ln 2}\|\Delta \mathbf{Q}\|_{F}^{2} \\
& -\frac{\kappa_{r}\|\Delta \mathbf{Q}\|_{F}}{\ln 2\left(1+\kappa_{r}\|\Delta \mathbf{Q}\|_{F}\right)} \operatorname{Trace}\left(\mathbf{I}_{m}-\frac{1}{\kappa_{r}} \boldsymbol{\Sigma}\right) .
\end{aligned}
$$

Remark 1: It is clear from (6) and (7) that under the small perturbation assumption, the degradation in capacity $C_{\mathrm{opt}}-C$ is dependent on $\|\Delta \mathbf{Q}\|_{F}$. With $\|\Delta \mathbf{Q}\|_{F} \rightarrow 0$, both the bounds approach $C_{\mathrm{opt}}$, and hence, $C \rightarrow C_{\mathrm{opt}}$. As a result, degradation can be minimized by minimizing $\|\Delta \mathrm{Q}\|_{F}$. This observation is used in later sections for the design of VQs.

Remark 2: When $m=2$, the only approximation used to obtain $(6)$ is $\ln (1-x) \approx-x$. For $m>2$, apart from this logarithmic approximation, the fact that $\left(\kappa_{r}^{2} / 2\right)\|\Delta \mathbf{Q}\|_{F}^{2}$ dominates all the other terms in the expansion of $\log \operatorname{det}\left[\mathbf{I}_{m}+\kappa_{r} \mathbf{V}^{*} \boldsymbol{\Delta} \mathbf{Q V}\right]$ is also used to obtain (6).

2) Analysis for Arbitrary Perturbations Based on Rank Criterion: We now study the variations in $C$ for arbitrary perturbations based on rank $\left(\widehat{\mathbf{Q}}_{\mathrm{opt}}\right)$. Let $\mathcal{Q}$ be the set of all $m \times m$ positive (semi) definite matrices with trace $P$. Let $\left\{\mathcal{E}_{j}\right\}_{j=1}^{m}$ be a partition of $\mathcal{Q}$ such that $\mathbf{Q} \in \mathcal{E}_{j}$ if $\operatorname{rank}(\mathbf{Q})=j$. Given the channel $\mathbf{H}$, we obtain upper and lower bounds on $C$ over every partition $\mathcal{E}_{j}$. This exercise is aimed at studying the extent of variation in $C$ due to a perturbed correlation matrix based on rank criterion. We highlight a few properties and applications of some of the bounds derived.

Upper Bounds on $C$ : For a given $\mathbf{H}$ and $j \in[1, m]$, we maximize $C$ over all $\widehat{\mathbf{Q}}_{\mathrm{opt}} \in \mathcal{E}_{j}$. The maximization is undertaken in Propositions 1 and 2, respectively, for $s \leq r$ and $s>r$.

Proposition 1: For a given channel, the optimal $\widehat{\mathbf{Q}}_{\mathrm{opt}}$ that maximizes $C$, subject to $\widehat{\mathbf{Q}}_{\mathrm{opt}} \in \mathcal{E}_{s}, s \leq r$, has the EVD $\widehat{\mathbf{Q}}_{\mathrm{opt}}=\mathbf{V} \boldsymbol{\Lambda}_{\widehat{\mathrm{Q}}_{\mathrm{opt}}} \mathbf{V}^{*}$, where

$$
\Lambda_{\widehat{Q}_{\mathrm{opt}}}(i, i)=-\frac{1}{\lambda_{i}(\mathbf{A})}+\frac{1}{s}\left(P+\sum_{j=1}^{s} \frac{1}{\lambda_{j}(\mathbf{A})}\right), i \in[1, s]
$$

and $\boldsymbol{\Lambda}_{\widehat{Q}_{\text {opt }}}(i, i)=0, i \in[s+1, m]$. The resulting upper bound on $C$, denoted by $C_{\mathrm{UB}}(s, r)$, is

$$
C_{\mathrm{UB}}(s, r)=\sum_{i=1}^{s} \log \left(\frac{\lambda_{i}(\mathbf{A})}{s}\left(P+\sum_{j=1}^{s} \frac{1}{\lambda_{j}(\mathbf{A})}\right)\right) .
$$

Proof: Noting that only the first $s$ eigenvalues of $\widehat{\mathbf{Q}}_{\mathrm{opt}}$ can be nonzero, the proof is similar to the one used to derive the optimal input correlation matrix in [1].

Corollary 1:

$$
C_{\mathrm{UB}}(s, r) \geq C_{\mathrm{UB}}(s-1, r) \cdots \geq C_{\mathrm{UB}}(1, r) \quad \forall s \leq r .
$$

Proof: See Appendix II

For $r<s$, the maximization of $C$ w.r.t. $\widehat{\mathbf{Q}}_{\mathrm{opt}}$ will not have a meaningful solution unless we impose the additional constraint, $\lambda_{i}\left(\widehat{\mathbf{Q}}_{\mathrm{opt}}\right) \geq \epsilon, i \in[1, s]$ for some small $\epsilon>0$. The reason is shown in Proposition 2.

Proposition 2: For a given channel, the optimal $\widehat{\mathbf{Q}}_{\mathrm{opt}}$ that maximizes $C$, subject to: 1$) \widehat{\mathbf{Q}}_{\mathrm{opt}} \in \mathcal{E}_{s}, s>r$; and 2) $\lambda_{i}\left(\widehat{\mathbf{Q}}_{\mathrm{opt}}\right) \geq \epsilon>0, i \in[1, s]$ has the EVD $\widehat{\mathbf{Q}}_{\mathrm{opt}}=\mathbf{V} \boldsymbol{\Lambda}_{\widehat{\mathrm{Q}}_{\mathrm{opt}}} \mathbf{V}^{*}$, where $\Lambda_{\widehat{Q}_{\mathrm{opt}}}(i, i)=\max \left(\mu-1 / \lambda_{i}(\mathbf{A}), \epsilon\right), i \in[1, r]$, $\boldsymbol{\Lambda}_{\widehat{Q}_{\mathrm{opt}}}(i, i) \stackrel{\boldsymbol{o p p t}^{\prime}}{=} \epsilon, i \in[r+1, s]$, and $\boldsymbol{\Lambda}_{\widehat{Q}_{\mathrm{opt}}}(i, i)=0, i \in[s+1, m]$ with $\sum_{i=1}^{r} \max \left(\mu-1 / \lambda_{i}(\mathbf{A}), \epsilon\right)=\stackrel{P}{P}-(s-r) \epsilon$.

Proof: The proof is similar to that used in Proposition 1. It can be shown further that

$\frac{-1}{\lambda_{t}(\mathbf{A})}+\frac{1}{t}\left(\sum_{i=1}^{t} \frac{1}{\lambda_{i}(\mathbf{A})}+P-(s-t) \epsilon\right)<\epsilon, t \in[r+1, s]$ thereby resulting in Proposition 2.

Lower Bounds on $C$ : For a given channel, we now minimize $C$ w.r.t. $\widehat{\mathbf{Q}}_{\text {opt }}$ for each $\mathcal{E}_{i}$. However, the solution to this problem is not meaningful unless the " $\epsilon$ " constraint $\lambda_{i}\left(\widehat{\mathbf{Q}}_{\mathrm{opt}}\right) \geq$ $\epsilon>0, i \in[1, s]$ is imposed. This is due to the following proposition.

Proposition 3: With $0<\epsilon \leq P / s$, the minimization of $C$ w.r.t. $\widehat{\mathbf{Q}}_{\mathrm{opt}} \in \mathcal{E}_{s}$ subject to $\lambda_{i}\left(\widehat{\mathbf{Q}}_{\mathrm{opt}}\right) \geq \epsilon>0, i \in[1, s]$ is equivalent to

$\min _{\widehat{\mathbf{Q}}_{\text {opt }}} C=\min _{\mathbf{W}} \log \operatorname{det}\left(\mathbf{I}_{m}+\boldsymbol{\Lambda}_{A} \mathbf{W} \boldsymbol{\Lambda}_{*} \mathbf{W}^{*}\right)$

s.t. W Orthonormal (10)

where $\Lambda_{*}$ is a diagonal matrix with entries $[P-(s-$ 1) $\epsilon, \epsilon, \ldots, \epsilon, 0 \ldots 0]$.

Proof: See Appendix III. 
Corollary 2: Under nontrivial conditions, the minimum achievable $C, C_{\text {SISO }}$, is

$$
C_{\mathrm{SISO}}=\log \left[1+P \lambda_{m}(\mathbf{A})\right]
$$

Proof: Follows from Proposition 3, and is omitted for brevity.

Remark: The capacity expression of a SISO additive white Gaussian channel [19] with input power $P$ and SNR amplification $\lambda_{m}(\mathbf{A})$ is similar to (11). Hence, the term $C_{\text {SISO }}$.

Applications of $C_{\mathrm{UB}}(s, r)$ : We now consider two applications of $C_{\mathrm{UB}}(s, r)$. The first application involves use of $C_{\mathrm{UB}}(s, r)$ to effect a rate-performance tradeoff. This is mentioned in Section IV. We now demonstrate the use of $C_{\mathrm{UB}}(m-1, m)$ in judging the reliability of feedback. Feedback is termed unreliable if $C \leq M I$, where $M I$ is the mutual information without CSIT and is given by $M I=\log \operatorname{det}\left[\mathbf{I}_{m}+(P / m) \mathbf{A}\right]$. We obtain sufficient conditions on the channel for which $C_{\mathrm{UB}}(m-1, m) \leq M I$. This, in turn, implies that any $\widehat{\mathbf{Q}}_{\text {opt }} \notin \mathcal{E}_{m}$ is unreliable.

Proposition 4: If the following condition holds true, then $C_{\mathrm{UB}}(m-1, m) \leq M I$ :

$$
\frac{1}{\lambda_{i}(\mathbf{A})} \leq \frac{1}{\lambda_{i+1}(\mathbf{A})} \leq \frac{1}{i}\left(\frac{P}{m}+\sum_{j=1}^{i} \frac{1}{\lambda_{j}(\mathbf{A})}\right)
$$

Proof: Let $\mathbf{Z}$ be the optimal correlation matrix achieving $C_{\mathrm{UB}}(m-1, m)$. Let $\underline{x}$ and $\underline{y}$ be $m \times 1$ vectors such that $\underline{x}(i)=$ $1 / \lambda_{i}(\mathbf{A})+\lambda_{i}(\mathbf{Z})$ and $\underline{y}(i) \stackrel{=}{=} 1 / \lambda_{i}(\mathbf{A})+P / m$. It is clear that $\underline{x}(i)=\left(P+\sum_{j=1}^{m-1} \overline{1} / \lambda_{j}(\mathbf{A})\right) /(m-1)$ for $i \in[1, m-1]$ and $\underline{x}(m)=1 / \lambda_{m}(\mathbf{A})$. If (12) holds, then it can be shown that $r=m$ and $\underline{y}$ majorizes $\underline{x}$ [20]. Then, similar to the proof of Corollary 1, we have $\prod_{i=1}^{m} \underline{x}(i) \geq \prod_{i=1}^{m} \underline{y}(i)$, and $C_{\mathrm{UB}}(m-$ $1, m) \leq M I$ follows.

Remark: For channels satisfying (12), using Corollary 1, we see that any perturbation that changes the rank of the correlation matrix results in an unreliable feedback.

3) Example: For $m=2$, we see that all correlation matrices belonging to $\mathcal{E}_{1}$ result in $C \leq C_{\mathrm{UB}}(1,2)<M I$ when $1 / \lambda_{2}(\mathbf{A}) \leq 1 / \lambda_{1}(\mathbf{A})+P / 2$.

\section{B. Channel Information Feedback}

In this scheme, we consider feedback of information about the channel needed to obtain $\mathbf{Q}_{\text {opt }}$. To keep the discussion simple, we consider feedback of $\mathbf{H}$ or $\mathbf{A}$, though other quantities can also be used. We begin by simplifying bounds of (6) and (7). The results thus obtained are valid for arbitrary $m, n$. Specializing to the case of $n \geq m$ and a channel with distinct singular values, we proceed to obtain an exact expression for the loss in capacity. Part of the work on obtaining exact expressions for the loss in capacity is presented in [21].

Use of (6) and (7) in this context requires expressing $\mathbf{Q}_{\mathrm{opt}}$ as a function of $\mathbf{H}$ or $\mathbf{A}$. However, it is cumbersome to express $\mathbf{Q}_{\text {opt }}$ directly in terms of $\mathbf{H}$ or $\mathbf{A}$. We now obtain a quantity, $\widetilde{\mathbf{A}}$, that can be easily related to $\mathbf{Q}_{\text {opt }}$ and will be prominently used in the ensuing analysis. Let $[\mathbf{B}]_{p}$ denote the best rank- $p$ approximation of a matrix $\mathbf{B}$ in the Frobenius sense [18]. Then

$$
\begin{aligned}
\mathbf{Q}_{\mathrm{opt}} & =-\widetilde{\mathbf{A}}+\frac{\operatorname{Trace}(\widetilde{\mathbf{A}})}{m} \mathbf{I}_{m}+\frac{P}{m} \mathbf{I}_{m} \text { where } \\
\widetilde{\mathbf{A}} & =\left[\mathbf{A}^{\dagger}\right]_{r}+\frac{1}{r}\left(P+\sum_{j=1}^{r} \frac{1}{\lambda_{j}(\mathbf{A})}\right)\left(\mathbf{I}_{m}-\left[\mathbf{A}^{\dagger}\right]_{r}[\mathbf{A}]_{r}\right) .
\end{aligned}
$$

1) Bounds on $C$ : Let the perturbed channel information at transmitter yield $(\widetilde{\mathbf{A}+\boldsymbol{\Delta} \mathbf{A}})$ instead of $\widetilde{\mathbf{A}}$. Hence $\widehat{\mathbf{Q}}_{\mathrm{opt}}=-\mathbf{A}+\boldsymbol{\Delta} \mathbf{A}+(\operatorname{Trace}(\mathbf{A}+\boldsymbol{\Delta} \mathbf{A}) / m) \mathbf{I}_{m}+(P / m) \mathbf{I}_{m}$. Then

$$
\begin{aligned}
\Delta \mathbf{Q} & =\widetilde{\mathbf{A}}-(\widetilde{\mathbf{A}+\boldsymbol{\Delta}} \mathbf{A})-\frac{1}{m}[\operatorname{Trace}[\widetilde{\mathbf{A}}-(\mathbf{A + \Delta} \mathbf{A})]] \mathbf{I}_{m} \\
\|\Delta \mathbf{Q}\|_{F}^{2} & =\|\widetilde{\mathbf{A}}-(\widetilde{\mathbf{A}+\Delta} \mathbf{A})\|_{F}^{2}-\frac{1}{m}(\operatorname{Trace}(\widetilde{\mathbf{A}}-(\widetilde{\mathbf{A}+\Delta} \mathbf{A})))^{2} .
\end{aligned}
$$

Use of (16) in (6) and (7) yields bounds on $C$ in terms of $\widetilde{\mathbf{A}}$ and $(\mathbf{A}+\Delta \mathbf{A})$. Note that the bounds are not directly in terms of $\mathbf{A}$ or $\mathbf{H}$. However, the dependence of these bounds on $\widetilde{(\mathbf{A})}$ sets up a case for the feedback of $\widetilde{\mathbf{A}}$ itself. This exercise deferred to Section IV, where it is shown that feedback of $\widetilde{\mathbf{A}}$ using VQ is more effective than that of $\mathbf{H}$ or $\mathbf{A}$. We now consider the special case of $n \geq m$ and derive expressions, instead of bounds, for $C_{\text {opt }}-C$.

2) $n \geq m$ : Analysis for First-Order Perturbation: While small perturbations are generally defined to have small absolute values, first-order perturbations are those for which the first-order approximations of [22] are valid. Let $\mathbf{\Delta A}$ be the resultant perturbation ${ }^{1}$ in $\mathbf{A}$. We term $\Delta \mathbf{A}$ as a first-order perturbation in $\mathbf{A}$, if $\|\mathbf{\Delta} \mathbf{A}\|<\delta \min \left(\lambda_{m}(\mathbf{A}), \operatorname{gap}(\mathbf{A})\right)$, where $\operatorname{gap}(\mathbf{A})=\min _{i}\left(\lambda_{i}(\mathbf{A})-\lambda_{i+1}(\mathbf{A})\right)$. We use $\delta=0.1$, a value obtained by simulations for which the first-order approximations of eigenvalues and eigenvectors given in [22] are within $1 \%$ of the actual value. For the analysis in this subsection, we assume that $\mathbf{H}$ is full rank with distinct singular values.

Let $\left\{\widehat{\widehat{v}}_{i}\right\}_{i=1}^{m}$ be the eigenvectors of $\mathbf{A}+\mathbf{\Delta A},[\mathbf{V}+\Delta \mathbf{V}]=$ $\left[\underline{\widehat{v}}_{1}, \widehat{\hat{v}}_{2}, \ldots, \underline{\widehat{v}}_{m}\right]$ and $\mathbf{V}=\left[\underline{v}_{1}, \underline{v}_{2}, \ldots, \underline{v}_{m}\right]$. Since $\mathbf{A}$ is a Hermitian matrix with distinct eigenvalues, using first-order approximations [22], we have $\lambda_{i}(\mathbf{A}+\boldsymbol{\Delta} \mathbf{A})=\lambda_{i}(\mathbf{A})+\Delta \lambda_{i}(\mathbf{A})$ and $\widehat{\underline{v}}_{i}=\underline{v}_{i}+\Delta \underline{v}_{i}$, where

$$
\Delta \underline{v}_{i}=\sum_{j=1}^{m} \frac{\underline{v}_{j} \underline{v}_{j}^{*}[\Delta \mathbf{A}] \underline{v}_{i}}{\lambda_{i}(\mathbf{A})-\lambda_{j}(\mathbf{A})}, \Delta \lambda_{i}(\mathbf{A})=\underline{v}_{i}^{*}[\Delta \mathbf{A}] \underline{v}_{i} i \in[1, m]
$$

Since first-order approximations hold, we use $\lambda_{i}(\mathbf{A})+$ $\underline{v}_{i}^{*}[\Delta \mathbf{A}] \underline{v}_{i}$ instead of $\lambda_{i}(\mathbf{A}+\boldsymbol{\Delta} \mathbf{A})$ for finding $\lambda_{j}\left(\widehat{\mathbf{Q}}_{\mathrm{opt}}\right)$ by waterfilling. With slight abuse of notation, we let $s$ denote the number of nonzero $\lambda_{i}\left(\widehat{\mathbf{Q}}_{\mathrm{opt}}\right)$ 's obtained by the use of

${ }^{1}$ If $\mathbf{H}$ is fed back and $\Delta \mathbf{H}$ is the perturbation in $\mathbf{H}$, then $\Delta \mathbf{A}=\mathbf{H}^{*}[\Delta \mathbf{H}]+$ $[\Delta \mathbf{H}] * \mathbf{H}+[\Delta \mathbf{H}] *[\Delta \mathbf{H}]$. 
$\lambda_{i}(\mathbf{A})+\underline{v}_{i}^{*}[\boldsymbol{\Delta} \mathbf{A}] \underline{v}_{i}$ instead of $\lambda_{i}(\mathbf{A}+\Delta \mathbf{A})$ during waterfilling. Then

$$
\begin{aligned}
\lambda_{i}\left(\widehat{\mathbf{Q}}_{\mathrm{opt}}\right)= & \frac{-1}{\lambda_{i}(\mathbf{A})+\underline{v}_{i}^{*}[\mathbf{\Delta} \mathbf{A}] \underline{v}_{i}} \\
& +\frac{1}{s}\left[P+\sum_{j=1}^{s} \frac{1}{\lambda_{j}(\mathbf{A})+\underline{v}_{j}^{*}[\mathbf{\Delta} \mathbf{A}] \underline{v}_{j}}\right], \quad i \in[1, s]
\end{aligned}
$$

and $\lambda_{i}\left(\widehat{\mathbf{Q}}_{\mathrm{opt}}\right)=0, i \in[s+1, m][1]$. By construction, $\widehat{\mathbf{Q}}_{\mathrm{opt}}=$ $[\mathbf{V}+\Delta \mathbf{V}]^{*} \Lambda_{\widehat{Q}_{\text {opt }}}[\mathbf{V}+\Delta \mathbf{V}]$. Letting $\mathbf{Z}=\mathbf{V}^{*} \Delta \mathbf{V}$ and $\mathbf{W}=$ $\mathbf{V}^{*}(\mathbf{V}+\boldsymbol{\Delta} \mathbf{V}) \stackrel{\text { opt }}{=} \mathbf{I}_{m}+\mathbf{Z}$, (3) can be written as

$$
C=\log \operatorname{det}\left[\mathbf{I}_{m}+\boldsymbol{\Lambda}_{A} \mathbf{W} \boldsymbol{\Lambda}_{\widehat{Q}_{\mathrm{opt}}} \mathbf{W}^{*}\right] .
$$

Retaining the first-order terms, we have $\left(\mathbf{I}_{m}+\mathbf{Z}\right) \boldsymbol{\Lambda}_{\widehat{Q}_{\text {opt }}}\left(\mathbf{I}_{m}+\right.$ $\left.\mathbf{Z}^{*}\right) \approx \Lambda_{\widehat{Q}_{\text {opt }}}+\mathbf{Z} \Lambda_{\widehat{Q}_{\text {opt }}}+\Lambda_{\widehat{Q}_{\text {opt }}} \mathbf{Z}^{*}$ (using definition for neglecting $\left.\mathbf{Z} \Lambda_{\widehat{Q}_{\text {opt }}} \mathbf{Z}^{*}\right) .^{2}$ Let $\delta_{(i, j)}=\lambda_{i}(\mathbf{A})\left(\mathbf{Z} \Lambda_{\widehat{Q}_{\mathrm{opt}}}+\right.$ $\left.\Lambda_{\widehat{Q}_{\text {opt }}} \mathbf{Z}^{*}\right)(i, j)$. Using (17) to find columns of $\Delta \mathbf{V}$, it can be shown that

$$
\begin{aligned}
& \delta_{(i, j)} \\
& =\lambda_{i}(\mathbf{A})\left(\frac{\underline{v}_{i}^{*} \Delta \mathbf{A} \underline{v}_{j}}{\lambda_{j}(\mathbf{A})-\lambda_{i}(\mathbf{A})}\right)\left(\frac{\lambda_{j}(\mathbf{A}+\boldsymbol{\Delta} \mathbf{A})-\lambda_{i}(\mathbf{A}+\mathbf{\Delta} \mathbf{A})}{\lambda_{j}(\mathbf{A}+\mathbf{\Delta} \mathbf{A}) \lambda_{i}(\mathbf{A}+\mathbf{\Delta} \mathbf{A})}\right), \\
& i \neq j, \quad \delta_{(i, i)}=0, i, j \leq s . \quad \text { (19) }
\end{aligned}
$$

For $i \leq s, j>s$, it can be shown that

$$
\begin{aligned}
\left|\delta_{(i, j)}\right| \leq \lambda_{i}(\mathbf{A})( & \left.\frac{\left|\underline{v}_{i}^{*} \mathbf{\Delta} \mathbf{A} \underline{v}_{j}\right|}{\lambda_{i}(\mathbf{A})-\lambda_{j}(\mathbf{A})}\right) \\
& \cdot\left(\frac{\lambda_{i}(\mathbf{A}+\boldsymbol{\Delta} \mathbf{A})-\lambda_{j}(\mathbf{A}+\boldsymbol{\Delta} \mathbf{A})}{\lambda_{i}(\mathbf{A}+\boldsymbol{\Delta} \mathbf{A}) \lambda_{j}(\mathbf{A}+\boldsymbol{\Delta} \mathbf{A})}\right) .
\end{aligned}
$$

A similar expression can be derived for $j \leq s, i>s$. Also note that $\delta_{i, j}=0, i, j \geq s+1$. Using $\lambda_{k}(\mathbf{A}+\boldsymbol{\Delta} \mathbf{A})=\lambda_{k}(\mathbf{A})+$ $\underline{v}_{k}^{*}[\mathbf{\Delta} \mathbf{A}] \underline{v}_{k}$ and noting that $\|\Delta \mathbf{A}\|<\lambda_{m}(\mathbf{A})$, we have

$$
\delta_{(i, j)} \approx 0 \quad \forall i, j \in[1, m] .
$$

${ }^{2}$ From the definition of first-order perturbations, it follows that the entries of $\mathbf{Z}$ are small. Hence, it is reasonable to neglect the entries of $\mathbf{Z} \boldsymbol{\Lambda}_{\widehat{Q}_{\text {opt }}} \mathbf{Z}^{*}$ in comparison with the corresponding diagonal entry of $\Lambda_{\widehat{Q}_{\mathrm{opt}}}$ for all $P$. Also note that $P$ is bounded for $s<m$, i.e., $P \lambda_{s+1}(\mathbf{A}+\Delta \mathbf{A})<s \forall s<m$. For larger $P, s$ increases to $m$. For $s=m, \Lambda_{\widehat{Q}_{\mathrm{opt}}}$ can be written as $\Sigma_{\widehat{Q}_{\mathrm{opt}}}+$ $(P / m) \mathbf{I}_{m}$, and an analysis independent of $P$ can be carried out by working with $\boldsymbol{\Lambda}_{A} \mathbf{W} \boldsymbol{\Sigma}_{\widehat{Q}_{\text {opt }}} \mathbf{W}^{*}$.
Equation (21) results in $\boldsymbol{\Lambda}_{A} \mathbf{W} \boldsymbol{\Lambda}_{\widehat{Q}_{\text {opt }}} \mathbf{W}^{*} \approx \boldsymbol{\Lambda}_{A} \boldsymbol{\Lambda}_{\widehat{Q}_{\text {opt }}}$. Using these results in (18), $C_{\text {pert }}$, the first-order approximation of $C$ is given by (22), shown at the bottom of the page.

3) Simulation Example: We present a numerical example to illustrate the analysis undertaken. Fig. 1 plots $C_{\text {pert }}, C$ [obtained from (3)] normalized w.r.t. $C_{\text {opt }}$ and the absolute difference $\left|C_{\text {pert }}-C\right|$ against the perturbation norm $\|\mathbf{\Delta} \mathbf{A}\|$, for a full-rank $4 \times 3$ channel chosen at random. The channel is scaled so that $\|\mathbf{H}\|=1$. In the experiment, $P=1, \mathbf{H}$ is fed back, and the perturbed CSI is $\mathbf{H}+\Delta \mathbf{H} . \Delta \mathbf{H}$ is chosen so that $\|\Delta \mathbf{A}\|$ is a first-order perturbation. A preset $\Delta \mathbf{H}$ is scaled to obtain different values of $\|\mathbf{\Delta} \mathbf{A}\|$. This plot and numerous simulations show that for first-order perturbations, $C_{\text {pert }}$ approximates $C$ very closely. Further, when 10000 realizations of first-order perturbations were generated for the above channel, it was seen that $r=s$ almost $96 \%$ of the time.

The above example and numerous other simulations have shown that first-order perturbations result in $r=s$ for most cases. This warrants a further study of perturbations resulting in $r=s$. This study is carried out in the following lemma.

Lemma 1: For first-order perturbations satisfying $r=s$, the loss in capacity is

$$
\begin{aligned}
C_{\mathrm{opt}}-C_{\mathrm{pert}} & =\frac{1}{2 \ln 2} \kappa_{s}^{2}\left(\sum_{i=1}^{s} \beta_{i}^{2}-\frac{1}{s}\left(\sum_{i=1}^{s} \beta_{i}\right)^{2}\right) \\
\beta_{i} & =\frac{\alpha_{i}}{\lambda_{i}(\mathbf{A})\left(\lambda_{i}(\mathbf{A})+\alpha_{i}\right)}
\end{aligned}
$$

where $\alpha_{i}=\underline{v}_{i}^{*}[\Delta \mathbf{A}] \underline{v}_{i}$.

Proof: Let $\chi_{i}=\lambda_{i}(\mathbf{A})\left(\lambda_{i}\left(\widehat{\mathbf{Q}}_{\mathrm{opt}}\right)-\lambda_{i}\left(\mathbf{Q}_{\mathrm{opt}}\right)\right) /(1+$ $\left.\lambda_{i}(\mathbf{A}) \lambda_{i}\left(\mathbf{Q}_{\mathrm{opt}}\right)\right)$. Then $C_{\mathrm{opt}}-C_{\mathrm{pert}}=-\sum_{i=1}^{s} \log \left(1+\chi_{i}\right)$. We shall now show that $\left|\chi_{i}\right| \ll 1, i \in[1, s]$. Define

$$
\begin{aligned}
\widehat{\nu}_{i, s}=\kappa_{s}\left(\frac{\|\mathbf{A}\|}{\lambda_{i}(\mathbf{A})\left(\lambda_{i}(\mathbf{A})-\|\mathbf{\Delta} \mathbf{A}\|\right)}\right. \\
\left.\quad+\frac{1}{s} \sum_{j=1}^{s} \frac{\|\mathbf{A} \mathbf{A}\|}{\lambda_{j}(\mathbf{A})\left(\lambda_{j}(\mathbf{A})-\|\mathbf{\Delta} \mathbf{A}\|\right)}\right), \quad i \in[1, s] .
\end{aligned}
$$

Using

$$
\begin{array}{r}
\lambda_{i}\left(\widehat{\mathbf{Q}}_{\mathrm{opt}}\right)=\frac{-1}{\lambda_{i}(\mathbf{A})+\alpha_{i}}+\frac{1}{s}\left[P+\sum_{j=1}^{s} \frac{1}{\lambda_{j}(\mathbf{A})+\alpha_{i}}\right] \\
\alpha_{i}=\underline{v}_{i}^{*}[\mathbf{\Delta} \mathbf{A}] \underline{v}_{i}
\end{array}
$$

$$
C_{\text {pert }}=\sum_{i=1}^{s} \log \left[1+\lambda_{i}(\mathbf{A}) \lambda_{i}\left(\widehat{\mathbf{Q}}_{\mathrm{opt}}\right)\right]=\sum_{i=1}^{s} \log \left(\frac{\underline{v}_{i}^{*}[\mathbf{\Delta} \mathbf{A}] \underline{v}_{i}}{\lambda_{i}(\mathbf{A})+\underline{v}_{i}^{*}[\mathbf{\Delta} \mathbf{A}] \underline{v}_{i}}+\frac{\lambda_{i}(\mathbf{A})}{s}\left[P+\sum_{j=1}^{s} \frac{1}{\lambda_{j}(\mathbf{A})+\underline{v}_{j}^{*}[\mathbf{\Delta} \mathbf{A}] \underline{v}_{j}}\right]\right)
$$


(a)

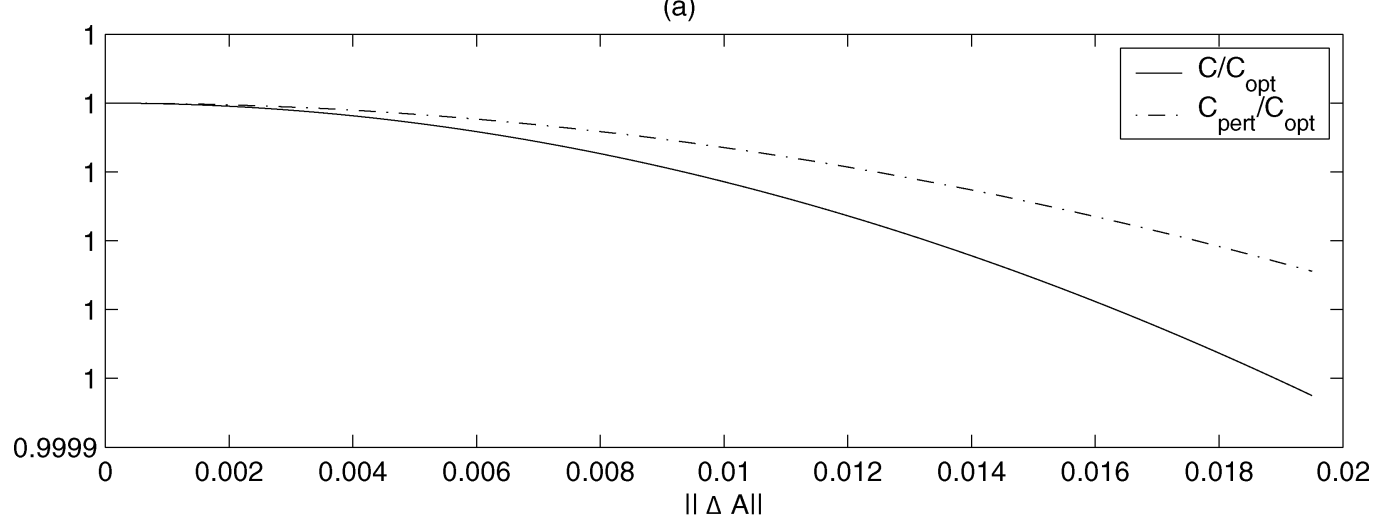

(b)

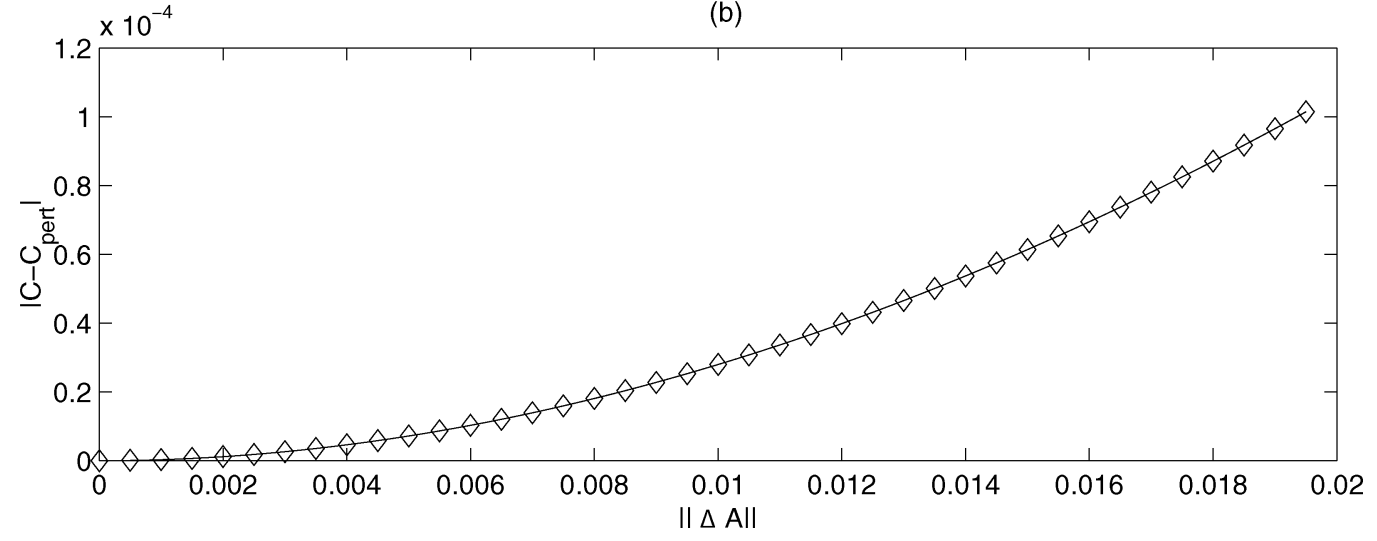

Fig. 1. (a) $C$ and $C_{\text {pert }}$ normalized w.r.t. $C_{\text {opt }}$. (b) $\left|C-C_{\text {pert }}\right|:\|\mathbf{H}\|=1, m=3, n=4, \lambda_{1}(\mathbf{A})=1, \lambda_{2}(\mathbf{A})=0.598, \lambda_{3}(\mathbf{A})=0.2, \max \|\Delta \mathbf{A}\|<0.02$, $C_{\mathrm{opt}}=5.6134$.

it is clear that $\left|\chi_{i}\right|<\widehat{\nu}_{i, s} i \in[1, s]$. Since rank $\left(\mathbf{Q}_{\mathrm{opt}}\right)=r=s$, $1 / \lambda_{i}(\mathbf{A}) \leq 1 / \kappa_{s}, i \in[1, s]$. Using this, we have

$$
\left|\chi_{i}\right|<\left(\frac{\|\Delta \mathbf{A}\|}{\left(\lambda_{i}(\mathbf{A})-\|\mathbf{\Delta} \mathbf{A}\|\right)}+\frac{1}{r} \sum_{j=1}^{r} \frac{\|\mathbf{\Delta} \mathbf{A}\|}{\left(\lambda_{j}(\mathbf{A})-\|\mathbf{\Delta} \mathbf{A}\|\right)}\right) .
$$

Then noting that $\|\mathbf{\Delta} \mathbf{A}\| \ll \lambda_{i}(\mathbf{A})$, we see that $\left|\chi_{i}\right| \ll 1, i \in$ $[1, s]$. Equation (23) follows by neglecting the higher order terms in the Taylor series expansion of $C_{\mathrm{opt}}-C_{\mathrm{pert}}$ and using the fact that $\sum_{i=1}^{s} \chi_{i}=0$.

Equation (23) requires information about the matrix $\Delta \mathbf{A}$. However, using $-\|\boldsymbol{\Delta} \mathbf{A}\| \leq \alpha_{i} \leq\|\boldsymbol{\Delta} \mathbf{A}\| \forall i$, we get an upper bound as

$C_{\mathrm{opt}}-C_{\mathrm{pert}}<\frac{\|\Delta \mathbf{A}\|^{2}}{2 \ln 2} \kappa_{s}^{2} \sum_{i=1}^{s}\left[\frac{1}{\lambda_{i}(\mathbf{A})\left(\lambda_{i}(\mathbf{A})-\|\mathbf{\Delta} \mathbf{A}\|\right)}\right]^{2}$.

Remark: Using unitary invariance of Frobenius norm [18], $\|\Delta \mathrm{Q}\|_{F}^{2}$ can be simplified to the right-hand side of (23) for first-order perturbations. However, the present analysis yields an exact expression for the loss in capacity, unlike the bounds derived earlier.

Effect of $P$ : The approximations for $\mathbf{V}+\boldsymbol{\Delta} \mathbf{V}$ and $\lambda_{i}(\mathbf{A}+$ $\Delta \mathbf{A}$ ) are independent of $P$. This shows that (22), as a firstorder approximation of $C$, holds for all values of $P$. However, variation of $C_{\mathrm{opt}}-C_{\text {pert }}$ with $P$ is difficult to analyze for $s<$ $m$, as $s$ depends on $P$. For large $P$, we have $r=s=m$, and (23) show that degradation falls as $P^{-2}$.

Effect of $\|\mathbf{\Delta} \mathbf{A}\|$ : Equation (24) and the prevalence of firstorder perturbations with $r=s$, shows that $C_{\mathrm{opt}}-C$ falls, approximately as $\|\Delta \mathbf{A}\|^{-2}$ for first-order perturbations.

Extensions to MIMO-OFDM Systems: The instantaneous mutual information for MIMO-orthogonal frequency-division multiplexing (OFDM) systems, obtained in [23] after neglecting overheads, is similar to (3). Hence, a similar perturbation analysis can be undertaken for MIMO-OFDM systems. This work is in progress.

In this section, we have studied the variations in $C$ due to fixed channel and unknown perturbation by deriving expressions and bounds. Norm-based and rank-based analyses were undertaken. We now employ the analysis to design a MIMO system with feedback for ergodic channels.

\section{DESIGN OF MIMO SySTEM With FEEDBACK FOR ERGODIC CHANNELS}

We consider a channel whose entries are modelled as i.i.d. circularly symmetric complex Gaussian random variables with zero mean and unit variance. The channel is considered ergodic [1], and the receiver is assumed to have perfect channel information. The ergodic capacity when the transmitter also has perfect CSI is $E\left(C_{\text {opt }}\right)$, where $C_{\text {opt }}$ is given in (2). In this section, we focus on a noiseless feedback supporting a maximum rate of $B$ 
bits per channel update. This rate limitation perturbs the information at the transmitter, and the resulting average mutual information is $E(C)$, where $C$ is given in (3). We consider design of VQs for feedback over such channels with an aim of minimizing the loss in ergodic capacity, $E\left(C_{\mathrm{opt}}-C\right)$. We also present $p a-$ rameter-based feedback, a scheme based on parametrization of feedback information. This scheme can be used to tradeoff the number of parameters fed back with the loss in capacity.

\section{A. VQ Design}

We begin by highlighting the ineffectiveness of the feedback scheme presented in [11] for the case of $n \geq m$. When $n \geq m$, it can be argued that rank $(\mathbf{A})=m$ with probability 1 for the ergodic channel mentioned above. This argument, coupled with an equal power allocation strategy, results in $\widehat{\mathbf{Q}}_{\mathrm{opt}}=(P / m) \mathbf{I}_{m}$ with probability 1 in [11] for $n \geq m$. However, it is obvious that the use of such a correlation matrix does not require any feedback. Thus, the feedback of eigenvectors in [11] is inefficient for $n \geq m$. This prompts us to consider schemes that feedback complete information about $\mathbf{Q}_{\text {opt }}$ to the transmitter in order to provide satisfactory performance. Towards this end, we consider the schemes proposed in Section III, and design VQs for the same.

1) VQ for Correlation Matrix Feedback: Assuming that the approximations used in (6) and (7) are valid, we consider the design of a VQ for feedback of $\mathbf{Q}_{\mathrm{opt}}$. It is clear from (6) and (7) that the loss in ergodic capacity, $E\left(C_{\mathrm{opt}}-C\right)$, can be minimized by minimizing $E\left(\kappa_{r}\|\Delta \mathbf{Q}\|_{F}\right)$. For ease of analysis, and also noting that $E\left(x^{2}\right) \geq E^{2}(x)$, we consider $E\left(\kappa_{r}^{2}\left(\|\Delta \mathbf{Q}\|_{F}^{2}\right)\right.$ as the cost function to be minimized in the design of VQs. The minimization is performed using the iterative codebook-based algorithm presented below.

\section{Algorithm:}

1) Nearest-Neighborhood Criterion $(N N C)$ : Let $N=2^{B}$. For a given set of code matrices $\left\{\widehat{\mathbf{Q}}_{k}\right\}_{k=1}^{N}, \widehat{\mathbf{Q}}_{k} \in \mathcal{Q}$, obtain optimum partition cells as

$$
\mathcal{Q}_{i}=\left\{\mathbf{Q}_{\mathrm{opt}} \in \mathcal{Q}:\left\|\mathbf{Q}_{\mathrm{opt}}-\widehat{\mathbf{Q}}_{k}\right\|_{F}^{2}>\left\|\mathbf{Q}_{\mathrm{opt}}-\widehat{\mathbf{Q}}_{i}\right\|_{F}^{2}, i \neq k\right\}
$$

where $\mathcal{Q}_{i}$ denotes the $i$ th partition cell of $\mathcal{Q}$ corresponding to $\widehat{\mathrm{Q}}_{i}$.

2) Centroid Criterion (CC): For a given partition $\mathcal{Q}_{i}$, obtain the centroid of $\mathcal{Q}_{i}$ as

$$
\begin{aligned}
\widehat{\mathbf{Q}}_{i} & =\arg \min _{\mathbf{Q} \in \mathcal{Q}} E\left[\kappa_{r}^{2}\left\|\mathbf{Q}_{\mathrm{opt}}-\mathbf{Q}\right\|_{F}^{2} \mid \mathbf{Q}_{\mathrm{opt}} \in \mathcal{Q}_{i}\right] \\
& =\frac{E\left[\kappa_{r}^{2} \mathbf{Q}_{\mathrm{opt}} \mid \mathbf{Q}_{\mathrm{opt}} \in \mathcal{Q}_{i}\right]}{E\left[\kappa_{r}^{2} \mid \mathbf{Q}_{\mathrm{opt}} \in \mathcal{Q}_{i}\right]} .
\end{aligned}
$$

The closed-form expression for the centroid given in (26) is derived in Appendix IV. Steps 1) and 2) are iterated until the reduction in the cost function is below a preset threshold [15].

Remark 1: It is not guaranteed that rank $\left(\widehat{\mathbf{Q}}_{i}\right)$ equals rank $\left(\mathbf{Q}_{\text {opt }}\right)$ even when $\mathbf{Q}_{\text {opt }} \in \mathcal{Q}_{i}$.

Remark 2: Similar to [11], the codebooks generated depend on $P$.
Remark 3: In the absence of statistical averages, sample averages are used in step 2) of the iteration. It can be easily argued that centroids thus obtained are valid correlation matrices.

Encoding: Given the codebook $\mathcal{C}_{Q}=\left\{\widehat{\mathbf{Q}}_{i}\right\}_{i=1}^{N}$, the receiver selects $\widehat{\mathbf{Q}}_{i}$ based on the channel $\mathbf{H}$ according to $\arg \min \widehat{\mathbf{Q}}_{j} \in \mathcal{C}_{Q}\left\|\mathbf{Q}_{\mathrm{opt}}-\widehat{\mathbf{Q}}_{j}\right\|_{F}^{2}$. The Hermitian structure of $\mathbf{Q}_{\mathrm{opt}}-\widehat{\mathbf{Q}}_{j}$ can be used to simplify the computation of $\left\|\mathbf{Q}_{\mathrm{opt}}-\widehat{\mathbf{Q}}_{j}\right\|_{F}^{2}$. The index $i$ is transmitted over the feedback link to the transmitter. The transmitter then shapes the input stream according to $\widehat{\mathbf{Q}}_{i}$ for transmission.

2) VQfor Channel Information Feedback: Information about $\left[\mathbf{A}^{\dagger}\right]_{r}, \mathbf{A}^{\dagger}, \widetilde{\mathbf{A}}, \mathbf{A}$ or $\mathbf{H}$ can be used to generate $\mathbf{Q}_{\mathrm{opt}}$ at the transmitter. Design of VQs for the feedback of any of these quantities is effective (and efficient) only when the cost function can be easily presented in terms of true and quantized entities. Towards this end, the equations derived in (16) and (23) set the stage for the quantization of $\widetilde{\mathbf{A}}$ and $\mathbf{A}$. The wider applicability of (6), (7) employing (16), and the fact that $\mathbf{Q}_{\text {opt }}$ relates to $\mathbf{A}$ through $\widetilde{\mathbf{A}}$ [(13)], further motivates us to consider quantization and feedback of $\widetilde{\mathbf{A}}$. Hence, we design a VQ for the feedback of $\widetilde{\mathbf{A}}$ using (16). Assuming that the bit budget is high enough, $E\left(C_{\mathrm{opt}}-C\right)$ is minimized by minimizing $E\left(\kappa_{r}^{2}\|\Delta \mathbf{Q}\|_{F}^{2}\right)$. However, the form for $\|\Delta \mathbf{Q}\|_{F}^{2}$ in (16) does not lead to an efficient iterative codebook design algorithm. It follows from (16) that $\|\mathbf{A} \mathbf{Q}\|_{F}^{2} \leq\|\widetilde{\mathbf{A}}-(\mathbf{A} \widetilde{+\Delta} \mathbf{A})\|_{F}^{2}$. This "quadratic" upper bound on $\|\Delta \mathrm{Q}\|_{F}^{2}$ yields a simple codebook design algorithm. Hence, we consider design of VQ for $\widetilde{\mathbf{A}}$ with $E\left[\kappa_{r}^{2}\|\widetilde{\mathbf{A}}-(\mathbf{A} \widetilde{+\Delta} \mathbf{A})\|_{F}^{2}\right]$ as the cost function.

Algorithm: Let $\mathcal{A}$ be the set of $m \times m$ positive definite matrices $\widetilde{\mathbf{A}}$, i.e., $\mathbf{Y} \in \mathcal{A} \Longleftrightarrow\left[-\mathbf{Y}+(\operatorname{Trace}(\mathbf{Y}) / m) \mathbf{I}_{m}+\right.$ $\left.(P / m) \mathbf{I}_{m}\right] \in \mathcal{Q}$.

1) $N N C$ : For a given set of code matrices $\left\{\mathbf{M}_{k}\right\}_{k=1}^{N}, \mathbf{M}_{k} \in$ $\mathcal{A}$, obtain optimum partition cells as

$$
\mathcal{A}_{i}=\left\{\widetilde{\mathbf{A}} \in \mathcal{A}:\left\|\widetilde{\mathbf{A}}-\mathbf{M}_{k}\right\|_{F}^{2}>\left\|\widetilde{\mathbf{A}}-\mathbf{M}_{i}\right\|_{F}^{2}, \quad i \neq k\right\}
$$

where $\mathcal{A}_{i}$ denotes the $i$ th partition cell of $\mathcal{A}$ corresponding to $\mathbf{M}_{i}$.

2) $C C$ : For a given partition $\mathcal{A}_{i}$, obtain the centroid of $\mathcal{A}_{i}$ as

$\mathbf{M}_{i}=\arg \min _{\mathbf{M} \in \mathcal{A}} E\left[\kappa_{r}^{2}\|\widetilde{\mathbf{A}}-\mathbf{M}\|_{F}^{2} \mid \widetilde{\mathbf{A}} \in \mathcal{A}_{i}\right]=\frac{E\left[\kappa_{r}^{2} \widetilde{\mathbf{A}} \mid \widetilde{\mathbf{A}} \in \mathcal{A}_{i}\right]}{E\left[\kappa_{r}^{2} \mid \widetilde{\mathbf{A}} \in \mathcal{A}_{i}\right]}$.

The closed-form expression for the centroid given in (28) is derived in Appendix IV. Steps 1) and 2) are iterated until the reduction in the cost function is below a threshold.

Remark 1: The quantizer design uses $\widetilde{\mathbf{A}}$, which, in general, contains information that is discarded during the generation of $\mathrm{Q}_{\mathrm{opt}}$. This is because the design is based on an upper bound of $\|\Delta \mathrm{Q}\|_{F}^{2}$.

Remark 2: The codebooks depend on $P$ as $\mathcal{A}$ depends on $P$.

Remark 3: In the absence of statistical averages, sample averages are used in step 2) of the iteration. It can be easily argued that centroids thus obtained yield valid correlation matrices. 
TABLE I

AVERAge Mutual INFormation FOR A $2 \times 2$ CHANNEL WITH $P=1, E(M I)=V Q_{V}=0.8096$

\begin{tabular}{c|c|c}
\hline Bits & $V Q_{Q}$ & $V Q_{\widetilde{A}}$ \\
\hline 1 & 0.8555 & 0.8535 \\
\hline 2 & 0.9084 & 0.9041 \\
\hline 3 & 0.9394 & 0.9394 \\
\hline 4 & 0.9700 & 0.9601 \\
\hline 5 & 0.9810 & 0.9729 \\
\hline 6 & 0.9887 & 0.9824 \\
\hline 7 & 0.9934 & 0.9877 \\
\hline 8 & 0.9962 & 0.9915 \\
\hline 9 & 0.9978 & 0.9943 \\
\hline 10 & 0.9988 & 0.9968 \\
\hline
\end{tabular}

TABLE II

AVERAGe Mutual INFORMATION FOR A $3 \times 4$ ChANNEL WITH $P=1, E(M I)=0.7190$

\begin{tabular}{c|c|c|c}
\hline Bits & $V Q_{Q}$ & $V Q_{V}$ & $V Q_{\widetilde{A}}$ \\
\hline 1 & 0.7337 & 0.7415 & 0.6902 \\
\hline 2 & 0.7508 & 0.7786 & 0.7459 \\
\hline 3 & 0.7683 & 0.7966 & 0.7641 \\
\hline 4 & 0.7936 & 0.8135 & 0.7914 \\
\hline 5 & 0.8191 & 0.8245 & 0.8193 \\
\hline 6 & 0.8417 & 0.8338 & 0.8434 \\
\hline 7 & 0.8612 & 0.8421 & 0.8611 \\
\hline 8 & 0.8781 & 0.8474 & 0.8789 \\
\hline 9 & 0.8934 & 0.8518 & 0.8936 \\
\hline 10 & 0.9067 & 0.8552 & 0.9076 \\
\hline
\end{tabular}

Encoding: Given the codebook $\mathcal{C}_{A}=\left\{\mathbf{M}_{i}\right\}_{i=1}^{N}$, the receiver selects $\mathbf{M}_{i}$ based on $\widetilde{\mathbf{A}}$ according to $\arg \min _{\mathbf{M}_{j} \in \mathcal{C}_{A}} \| \widetilde{\mathbf{A}}-$ $\mathbf{M}_{j} \|_{F}^{2}$. The index $i$ is fed back to the transmitter. The transmitter then obtains $\widehat{\mathbf{Q}}_{\text {opt }}$ by using $\mathbf{M}_{i}$ instead of $\widetilde{\mathbf{A}}$ in (13) for shaping the input stream.

3) Performance of VQs: Tables I and II present the performance of the proposed VQs for $2 \times 2$ and $3 \times 4$ channels, respectively, under various bit budgets. $P$ is chosen as 1 . The quantizer designed in [11] is also presented for comparison. In these tables, $E(M I)$ denotes the average mutual information with no feedback and equal power allocation, while $V Q_{V}$ denotes the average mutual information achieved by [11]. $V Q_{Q}$ and $V Q_{\widetilde{A}}$, respectively, denote the average mutual information $(E(C))$ with covariance matrix feedback and channel information feedback. All the quantities are normalized with respect to $E\left(C_{\text {opt }}\right)$, the ergodic capacity.

Remark 1: In Table I, $V Q_{V}=E(M I)$ and is only $80 \%$ of the ergodic capacity. However, using the proposed VQs, 96\%-97\% of the ergodic capacity is attained with as low as $B=4$ bits. Large improvements over [11] are also seen from simulations for different $m, n$ with $m \leq n$.

Remark 2: For $m \geq n$, it is known a priori that rank $\left(\mathbf{Q}_{\mathrm{opt}}\right) \leq n$. The proposed quantizers do not exploit this
TABLE III

Average Mutual Information for a $2 \times 2$ ChanNel With $P=1$, $E(M I)=0.8096, \mathbf{H}, \mathbf{A}$ ARE FED BACK, VQ IS EMPLOYED WITH HEURISTIC COST FUNCTIONS

\begin{tabular}{c|c|c}
\hline Bits & $V Q_{H}$ & $V Q_{A}$ \\
\hline 1 & 0.6950 & 0.8112 \\
\hline 2 & 0.7178 & 0.8277 \\
\hline 3 & 0.7740 & 0.8553 \\
\hline 4 & 0.8003 & 0.8846 \\
\hline 5 & 0.8432 & 0.9123 \\
\hline 6 & 0.8768 & 0.9328 \\
\hline 7 & 0.8956 & 0.9560 \\
\hline 8 & 0.9079 & 0.9704 \\
\hline 9 & 0.9245 & 0.9838 \\
\hline 10 & 0.9344 & 0.9911 \\
\hline
\end{tabular}

information, unlike the design of [11], to improve their resolution. For the proposed quantizers with small values of $B$, the resulting perturbations are not small enough to validate the approximations used in (6) and (7). Hence, at low feedback rates, performance of the proposed quantizers are not satisfactory. However, for larger values of $B$, the approximations used are validated, and the performance of the proposed quantizers improve. This is highlighted for $m=4, n=3$ in Table II, where the proposed quantizers outperform the design of [11] for $B \geq 6$. This improvement is due to the complete nature of feedback.

These results along with other simulations show that significant gains in mutual information can be obtained by feedback of complete information over a limited-rate channel.

4) On Feedback of $\mathbf{H}$ or $\mathbf{A}$ : Clearly, the design of VQs for $\mathbf{H}$ or $\mathbf{A}$ through $\mathbf{Q}_{\text {opt }}$ or $\widetilde{\mathbf{A}}$ is inefficient, and does not yield any performance benefits. The obvious cost function $E\left[\|\Delta \mathbf{H}\|_{F}^{2}\right]$ (or $E\left[\|\boldsymbol{\Delta} \mathbf{A}\|_{F}^{2}\right]$ ) allows a computationally efficient VQ design for feedback of $\mathbf{H}$ (or $\mathbf{A}$ ) similar to the designs presented earlier. Here $\boldsymbol{\Delta} \mathbf{H}(\boldsymbol{\Delta} \mathbf{A})$ is the perturbation in $\mathbf{H}(\mathbf{A})$. The performance of VQs for $\mathbf{H}$ and $\mathbf{A}$ minimizing these heuristic cost functions is presented in Table III for a $2 \times 2$ channel with $P=1$. For these VQ designs, $\mathrm{VQ}_{H}$ and $\mathrm{VQ}_{A}$ denote the average mutual information for feedback of $\mathbf{H}$ and $\mathbf{A}$, respectively. The unsatisfactory performance of the VQs designed using a heuristic cost function is clearly seen by comparing Tables I and III. This result highlights the efficiency of feedback of $\mathbf{Q}_{\mathrm{opt}}$ and $\widetilde{\mathbf{A}}$ over limited-rate channels.

\section{B. Parameter-Based Feedback}

We conclude by presenting the non-codebook parameter-based feedback scheme for correlation matrices. This scheme is based on the parametrization of eigenvalues and eigenvectors. Extension to the feedback of $\widetilde{\mathbf{A}}$ is also provided. We denote $r=\operatorname{rank}\left(\mathbf{Q}_{\mathrm{opt}}\right)$ throughout this discussion.

Feedback of Eigenvectors: An arbitrary $t \times p$ orthonormal matrix can be parameterized by $2 p t-p^{2}$ real quantities using Givens rotations [17]. We use this parametrization for the feedback of eigenvectors of $\mathbf{Q}_{\mathrm{opt}}$ ( or $\widetilde{\mathbf{A}}$ ). The number of parameters would then be $2 m r-r^{2}$ as rank $\left(\mathbf{Q}_{\mathrm{opt}}\right)=r$. 
Feedback of Eigenvalues: We consider the feedback of nonzero $\left\{\lambda_{i}\left(\mathbf{Q}_{\mathrm{opt}}\right)\right\}$ 's. The ordering and sum constraint of nonzero $\left\{\lambda_{i}\left(\mathbf{Q}_{\mathrm{opt}}\right)\right\}$ is exploited in the parametrization presented below

$$
\begin{aligned}
\lambda_{r}\left(\mathbf{Q}_{\mathrm{opt}}\right) & =\frac{P}{r} \cos ^{2} \alpha_{1}, \lambda_{k}\left(\mathbf{Q}_{\mathrm{opt}}\right)-\lambda_{k+1}\left(\mathbf{Q}_{\mathrm{opt}}\right) \\
& =\frac{P}{k} \prod_{i=1}^{r-k} \sin ^{2} \alpha_{i} \cos ^{2} \alpha_{r-k+1}, k \in[r-1,2] \\
\lambda_{1}\left(\mathbf{Q}_{\mathrm{opt}}\right)-\lambda_{2}\left(\mathbf{Q}_{\mathrm{opt}}\right) & =P \prod_{i=1}^{r-1} \sin ^{2} \alpha_{i} .
\end{aligned}
$$

The parameters $\left\{\alpha_{k}\right\}_{k=1}^{r-1}$ are transmitted on the feedback link. The proposed parametrization maintains the ordering and the sum constraint of the eigenvalues even in the presence of feedback errors. Modifications are needed for feedback of $\lambda_{i}(\widetilde{\mathbf{A}})$ due to the absence of a trace constraint. Let $t_{A}=\operatorname{Trace}(\widetilde{\mathbf{A}})$. Then the eigenvalue feedback is effected by employing (29) with $t_{A}$ instead of $P$ and $r=m$. The parameters fed back now include $t_{A}$ (or $\sqrt{t_{A}}$ ) along with $\left\{\alpha_{i}\right\}$.

Knowledge of $r$ at Transmitter: Let $N_{p}$ denote the total number of parameters used in the feedback of $\mathbf{Q}_{\text {opt }}$. When rank $\left(\mathbf{Q}_{\mathrm{opt}}\right)=r, N_{p}=2 m r-r^{2}+r-1$. Using this formula, the transmitter can obtain the value of $r$ by counting the number of parameters fed back. Thus, $r$ need not be fed back explicitly.

Tradeoff Between $N_{p}$ and Loss in Capacity: In the above scheme, feedback of $\widehat{\mathbf{Q}}_{\text {opt }}$ with rank $\left(\widehat{\mathbf{Q}}_{\mathrm{opt}}\right)<r$ reduces $N_{p}$. However, feedback of $\widehat{\mathbf{Q}}_{\mathrm{opt}}$ brings about a loss in capacity. Use of Corollary 1 then highlights a tradeoff between $N_{p}$ and the loss in capacity. If the feedback errors are small, the loss in capacity can be (approximately) minimized by choosing $\widehat{\mathbf{Q}}_{\mathrm{opt}}$, as in Proposition 1. Further, the above tradeoff is essentially a rate-performance tradeoff, since $N_{p}$ relates to the feedback rate.

\section{CONCLUSIONS}

In this paper, we address the issue of loss in achievable rates due to perturbed information at the transmitter in a MIMO system. Two feedback schemes, correlation matrix feedback and channel information feedback, are proposed, and their performance is studied based on analytical results. Using these results, VQs are designed for correlation matrix/channel information feedback to minimize the loss in ergodic capacity. Performance of these quantizers are studied, and gains obtained by quantized feedback are tabulated. The performance of a system that uses a channel matrix for feedback over a rate-constrained link is shown to be unsatisfactory.

\section{APPENDIX I}

Proof of (5): Since rank $\left(\mathbf{Q}_{\mathrm{opt}}\right)=r$, it can be deduced that $1 / \lambda_{r+1}(\mathbf{A})>(1 / r)\left(P+\sum_{j=1}^{r} 1 / \lambda_{j}(\mathbf{A})\right)=1 / \kappa_{r}$. Using $1 / \lambda_{k}(\mathbf{A}) \geq 1 / \lambda_{r+1}(\mathbf{A})>1 / \kappa_{r}, k \geq r+1$, it can be shown that $\boldsymbol{\Theta}=\kappa_{r} \mathbf{I}_{m}-\boldsymbol{\Sigma}$ is a positive semidefinite matrix. Let $\boldsymbol{\Psi}=\left[\mathbf{I}_{m}+\boldsymbol{\Sigma} \mathbf{V}^{*}[\Delta \mathbf{Q}] \mathbf{V}\right], \boldsymbol{\Psi}_{D}=\mathbf{I}_{m}-\kappa_{r} \boldsymbol{\Lambda}_{\mathbf{Q}_{\mathrm{opt}}}$, and $\Psi_{X}=\mathbf{V}^{*} \widehat{\mathbf{Q}}_{\mathrm{opt}} \mathbf{V}$. Using $\boldsymbol{\Sigma} \boldsymbol{\Lambda}_{\mathbf{Q}_{\mathrm{opt}}}=\kappa_{r} \boldsymbol{\Lambda}_{\mathbf{Q}_{\mathrm{opt}}}$, we have $\operatorname{det} \boldsymbol{\Psi}=\operatorname{det}\left[\boldsymbol{\Psi}_{D}+\kappa_{r} \Psi_{X}-\boldsymbol{\Theta} \Psi_{X}\right]$. Using the expression for $\kappa_{r}$, it can be deduced that $\boldsymbol{\Psi}_{D}>\mathbf{0}$. As a result, $\Psi_{D}^{-1}$ exits, and we have $\operatorname{det} \boldsymbol{\Psi}=\operatorname{det}\left[\boldsymbol{\Psi}_{D}\right] \operatorname{det}\left[\mathbf{I}_{m}+\kappa_{r} \boldsymbol{\Psi}_{D}^{-1} \boldsymbol{\Psi}_{X}-\boldsymbol{\Psi}_{D}^{-1} \boldsymbol{\Theta} \boldsymbol{\Psi}_{X}\right]$. Using $\operatorname{det}(\mathbf{I}+\mathbf{A B})=\operatorname{det}(\mathbf{I}+\mathbf{B A})$ and the positive (semi)-definiteness of $\Psi_{X}$, we have

$\operatorname{det} \boldsymbol{\Psi}=\operatorname{det}\left[\boldsymbol{\Psi}_{D}\right] \operatorname{det}\left[\mathbf{I}_{m}+\kappa_{r} \boldsymbol{\Psi}_{X}^{1 / 2} \boldsymbol{\Psi}_{D}^{-1} \boldsymbol{\Psi}_{X}^{1 / 2}-\boldsymbol{\Psi}_{X}^{1 / 2} \boldsymbol{\Psi}_{D}^{-1} \boldsymbol{\Theta} \boldsymbol{\Psi}_{X}^{1 / 2}\right]$

where $\Psi_{X}^{1 / 2}$ is a positive semidefinite matrix with $\Psi_{X}^{1 / 2} \boldsymbol{\Psi}_{X}^{1 / 2}=$ $\Psi_{X}$ [18]. Since the involved matrices are positive (semi)-definite, it can be argued that

$$
\begin{aligned}
\operatorname{det}\left[\mathbf{I}_{m}+\kappa_{r} \boldsymbol{\Psi}_{X}^{1 / 2} \boldsymbol{\Psi}_{D}^{-1} \Psi_{X}^{1 / 2}-\right. & \left.\boldsymbol{\Psi}_{X}^{1 / 2} \boldsymbol{\Psi}_{D}^{-1} \boldsymbol{\Theta} \boldsymbol{\Psi}_{X}^{1 / 2}\right] \\
& \leq \operatorname{det}\left[\mathbf{I}_{m}+\kappa_{r} \Psi_{X}^{1 / 2} \Psi_{D}^{-1} \Psi_{X}^{1 / 2}\right]
\end{aligned}
$$

Using this inequality, we have $\operatorname{det} \Psi \leq \operatorname{det}\left[\boldsymbol{\Psi}_{D}\right] \operatorname{det}\left[\mathbf{I}_{m}+\right.$ $\left.\kappa_{r} \Psi_{X}^{1 / 2} \Psi_{D}^{-1} \boldsymbol{\Psi}_{X}^{1 / 2}\right]$. Equation (5) then follows by noting that $\operatorname{det}\left[\boldsymbol{\Psi}_{D}\right] \operatorname{det}\left[\mathbf{I}_{m}+\kappa_{r} \boldsymbol{\Psi}_{X}^{1 / 2} \boldsymbol{\Psi}_{D}^{-1} \boldsymbol{\Psi}_{X}^{1 / 2}\right]=\operatorname{det}\left[\mathbf{I}_{m}+\kappa_{r}[\Delta \mathbf{Q}]\right]$

Simplification of $\log \operatorname{det}\left[\mathbf{I}_{m}+\kappa_{r}[\Delta \mathbf{Q}]\right]$ : Let $\left\{\lambda_{i}(\boldsymbol{\Delta} \mathbf{Q})\right\}_{i=1}^{m}$ be the eigenvalues of $\Delta \mathbf{Q}$. On expansion, we have the equation shown at the bottom of the page, where $R_{r}$ denotes other symmetric products. Since $\operatorname{Trace}(\boldsymbol{\Delta} \mathbf{Q})=0$, it can be shown that

$$
\sum_{i=1, j<i}^{m} \lambda_{i}(\boldsymbol{\Delta} \mathbf{Q}) \lambda_{j}(\boldsymbol{\Delta} \mathbf{Q})=-\frac{1}{2} \sum_{i=1}^{m} \lambda_{i}^{2}(\boldsymbol{\Delta} \mathbf{Q})=-\frac{1}{2} \operatorname{Trace}(\boldsymbol{\Delta} \mathbf{Q})^{2} .
$$

Further, it can be verified that

$$
\begin{aligned}
\left(\sum_{i=1}^{k} \lambda_{i}(\mathbf{\Delta} \mathbf{Q})\right)^{3}= & \left(\sum_{i=1}^{k-1} \lambda_{i}(\mathbf{\Delta} \mathbf{Q})\right)^{3}+\lambda_{k}^{3}(\Delta \mathbf{Q}) \\
& -3 \lambda_{k}(\Delta \mathbf{Q}) \sum_{i=1}^{k-1} \lambda_{i}(\Delta \mathbf{Q})\left[\sum_{i=k+1}^{m} \lambda_{i}(\mathbf{\Delta} \mathbf{Q})\right]
\end{aligned}
$$

$$
\begin{aligned}
& \log \operatorname{det}\left[\mathbf{I}_{m}+\kappa_{r}[\mathbf{\Delta} \mathbf{Q}]\right] \\
& =\log \left[1+\kappa_{r}\left(\sum_{i=1}^{m} \lambda_{i}(\boldsymbol{\Delta} \mathbf{Q})\right)+\kappa_{r}^{2} \sum_{i=1, j<i}^{m} \lambda_{i}(\boldsymbol{\Delta} \mathbf{Q}) \lambda_{j}(\boldsymbol{\Delta} \mathbf{Q})+\kappa_{r}^{3} \sum_{i=1, j<i, k<j}^{m} \lambda_{i}(\boldsymbol{\Delta} \mathbf{Q}) \lambda_{j}(\Delta \mathbf{Q}) \lambda_{k}(\boldsymbol{\Delta} \mathbf{Q})+R_{r}\right]
\end{aligned}
$$


where we have used $\sum_{i=1}^{k} \lambda_{i}(\boldsymbol{\Delta} \mathbf{Q})=-\sum_{i=k+1}^{m} \lambda_{i}(\boldsymbol{\Delta} \mathbf{Q})$. Using this expression iteratively for $k=m, m-1, \ldots, 1$, we see that

$$
\begin{aligned}
\sum_{i=1, j<i, k<j}^{m} \lambda_{i}(\boldsymbol{\Delta} \mathbf{Q}) \lambda_{j}(\boldsymbol{\Delta} \mathbf{Q}) \lambda_{k}(\boldsymbol{\Delta} \mathbf{Q}) & =\frac{1}{3} \sum_{i=1}^{m} \lambda_{i}^{3}(\boldsymbol{\Delta} \mathbf{Q}) \\
& =\frac{1}{3} \operatorname{Trace}(\boldsymbol{\Delta} \mathbf{Q})^{3} .
\end{aligned}
$$

Use of these results in the previously mentioned expansion of $\log \operatorname{det}\left[\mathbf{I}_{m}+\kappa_{r}[\Delta \mathbf{Q}]\right]$ yields the required simplification.

Proof of (7): Note that

$$
\begin{aligned}
C= & C_{\mathrm{opt}}+\log \operatorname{det}\left[\mathbf{I}_{m}+\kappa_{r} \mathbf{V}^{*}[\boldsymbol{\Delta} \mathbf{Q}] \mathbf{V}\right] \\
& +\log \operatorname{det}\left\{\left[\mathbf{I}_{m}+\kappa_{r} \mathbf{V}^{*}[\boldsymbol{\Delta} \mathbf{Q}] \mathbf{V}\right]^{-1}\left[\mathbf{I}_{m}+\mathbf{\Sigma} \mathbf{V}^{*}[\boldsymbol{\Delta} \mathbf{Q}] \mathbf{V}\right]\right\} .
\end{aligned}
$$

Letting $\mathbf{Y}=\kappa_{r} \mathbf{V}^{*}[\boldsymbol{\Delta} \mathbf{Q}] \mathbf{V}, \boldsymbol{\Theta}=\mathbf{I}_{m}-\left(1 / \kappa_{r}\right) \boldsymbol{\Sigma}$ and using $\operatorname{det}(\mathbf{I}+\mathbf{A B})=\operatorname{det}(\mathbf{I}+\mathbf{B A})$ and $\mathbf{Y}\left(\mathbf{I}_{m}+\mathbf{Y}\right)^{-1}=\mathbf{I}_{m}-$ $\left(\mathbf{I}_{m}+\mathbf{Y}\right)^{-1}$, it can be shown that

$$
\begin{aligned}
\log \operatorname{det}\left\{\left[\mathbf{I}_{m}+\kappa_{r} \mathbf{V}^{*}[\Delta \mathbf{Q}] \mathbf{V}\right]^{-1}\left[\mathbf{I}_{m}+\boldsymbol{\Sigma} \mathbf{V}^{*}[\Delta \mathbf{Q}] \mathbf{V}\right\}\right] \\
=\log \operatorname{det}\left[\mathbf{I}_{m}-\left(\mathbf{I}_{m}-\left(\mathbf{I}_{m}+\mathbf{Y}\right)^{-1}\right) \mathbf{\Theta}\right] .
\end{aligned}
$$

Further, $\left(\mathbf{I}_{m}+\mathbf{Y}\right)^{-1} \geq 1 /\left(1+\|\mathbf{Y}\|_{F}\right) \mathbf{I}_{m}$. Since $\boldsymbol{\Theta} \geq 0, \boldsymbol{\Theta}^{1 / 2}$ exits, and it can be argued that $\mathbf{I}_{m}-\boldsymbol{\Theta}^{1 / 2}\left(\mathbf{I}_{m}-\left(\mathbf{I}_{m}+\mathbf{Y}\right)^{-1} \boldsymbol{\Theta}^{1 / 2} \geq\right.$ $\mathbf{I}_{m}-\|\mathbf{Y}\|_{F} /\left(1+\|\mathbf{Y}\|_{F}\right) \boldsymbol{\Theta}$. It then follows that

$$
\begin{aligned}
\operatorname{det}\left[\mathbf{I}_{m}-\boldsymbol{\Theta}^{1 / 2}\left(\mathbf{I}_{m}-\left(\mathbf{I}_{m}+\mathbf{Y}\right)^{-1}\right)\right. & \left.\boldsymbol{\Theta}^{1 / 2}\right] \\
& \geq \operatorname{det}\left[\mathbf{I}_{m}-\frac{\|\mathbf{Y}\|_{F}}{1+\|\mathbf{Y}\|_{F}} \boldsymbol{\Theta}\right]
\end{aligned}
$$

Using $\|\mathbf{Y}\|_{F}=\kappa_{r}\|\Delta \mathbf{Q}\|_{F}$ and $\operatorname{det}(\mathbf{I}+\mathbf{A B})=\operatorname{det}(\mathbf{I}+\mathbf{B A})$, we have

$$
\begin{aligned}
C \geq C_{\text {opt }}+\log \operatorname{det}\left[\mathbf{I}_{m}+\kappa_{r} \Delta \mathbf{Q}\right] & \\
& +\log \operatorname{det}\left[\mathbf{I}_{m}-\kappa_{r} \frac{\|\Delta \mathbf{Q}\|_{F}}{1+\kappa_{r}\|\Delta \mathbf{Q}\|_{F}} \boldsymbol{\Theta}\right] .
\end{aligned}
$$

\section{APPENDIX II}

\section{PROOF OF COROLLARY 1}

We shall prove $C_{\mathrm{UB}}(s, r) \geq C_{\mathrm{UB}}(s-1, r)$ and the rest of the inequality can be similarly deduced. Let $\left\{\alpha_{i}\right\}_{i=1}^{s}$ be the nonzero eigenvalues of $\widehat{\mathbf{Q}}_{\mathrm{opt}}$ achieving $C_{\mathrm{UB}}(s, r)$. Similarly, let $\left\{\beta_{i}\right\}_{i=1}^{s-1}$ be the nonzero eigenvalues of $\widehat{\mathbf{Q}}_{\mathrm{opt}}$ achieving $C_{\mathrm{UB}}(s-1, r)$. Letting $\underline{x}(i)=1 / \lambda_{i}(\mathbf{A})+\alpha_{i}=$ $(1 / s)\left(P+\sum_{j=1}^{s} 1 / \lambda_{j}(\mathbf{A})\right), i \in[1, s]$, let

$$
\underline{y}(i)=\frac{1}{\lambda_{i}(\mathbf{A})}+\beta_{i}=\frac{1}{s-1}\left(P+\sum_{j=1}^{s-1} \frac{1}{\lambda_{j}(\mathbf{A})}\right), i \in[1, s-1]
$$

and $y(s)=1 / \lambda_{s}(\mathbf{A})$. Since $\alpha_{s}>0,1 / \lambda_{s}(\mathbf{A}) \leq$ $\left(P+\sum_{j=1}^{s-1} 1 / \lambda_{j}(\mathbf{A})\right) /(s-1)$. Using these, it can be shown that $y(s) \leq \underline{x}(s)$ and hence, $y(i) \geq \underline{x}(i), i \in[1, s-1]$. It can than be shown that the vector $\underline{y}$ majorizes the vector $\underline{x}$ [20]. From the Schur concavity of the product [20], we have $\prod_{i=1}^{s} \underline{x}(i) \geq$ $\prod_{i=1}^{s} \underline{y}(i)$. Then, $\prod_{i=1}^{s} \lambda_{i}(\mathbf{A}) \underline{x}(i) \geq \prod_{i=1}^{s} \lambda_{i}(\mathbf{A}) \underline{y}(i)$, and hence, $C_{\mathrm{UB}}(s, r) \geq C_{\mathrm{UB}}(s-1, r)$ follows.

\section{APPENDIX III \\ PROOF OF PROPOSITION 3}

Let $\widehat{\mathbf{Q}}_{\mathrm{opt}}=\widehat{\mathbf{V}} \boldsymbol{\Lambda}_{\widehat{Q}_{\mathrm{opt}}} \widehat{\mathbf{V}}^{*}$ be the EVD of $\widehat{\mathbf{Q}}_{\mathrm{opt}}$, and let $\mathbf{W}=$ $\mathbf{V}^{*} \widehat{\mathbf{V}}$. Let $C\left(\mathbf{W}, \boldsymbol{\Lambda}_{\widehat{Q}_{\text {opt }}}\right)=\log \operatorname{det}\left[\mathbf{I}_{m}+\boldsymbol{\Lambda}_{A} \mathbf{W} \boldsymbol{\Lambda}_{\widehat{Q}_{\text {opt }}} \mathbf{W}^{*}\right]$. The minimization problem can be written as $\min _{\mathbf{W}} C(\mathbf{W})$ subject to $\mathbf{W}^{*} \mathbf{W}=\mathbf{I}_{m}$. Here $C(\mathbf{W})=\min _{\boldsymbol{\Lambda}_{\widehat{Q}_{\mathrm{opt}}}} C\left(\mathbf{W}, \boldsymbol{\Lambda}_{\widehat{Q}_{\mathrm{opt}}}\right)$ subject to: 1) $\boldsymbol{\Lambda}_{\widehat{Q}_{\mathrm{opt}}}(i, i) \geq \epsilon, i \in[1, s]$; 2) $\boldsymbol{\Lambda}_{\widehat{Q}_{\mathrm{opt}}}(i, i)=$ $0, i \in[s+1, m]$; 3) $\sum_{i=1}^{s} \Lambda_{\widehat{Q}_{\mathrm{opt}}}(i, i)=P$; and 4) $\Lambda_{\widehat{Q}_{\text {opt }}}(1,1) \geq \Lambda_{\widehat{Q}_{\text {opt }}}(2,2), \ldots, \geq \Lambda_{\widehat{Q}_{\text {opt }}}(s, s)$. The minimization of $C\left(\mathbf{W}, \Lambda_{\widehat{Q}_{\text {opt }}}\right)$ with the associated constraints is a concave optimization problem for every orthonormal $\mathbf{W}$ [18]. The minima for such a problem occurs at the extreme points of the constraint set [24]. Since $\epsilon \leq P / s$, the extreme points of the constraint set are $\boldsymbol{\Lambda}_{*}=\operatorname{diag}(P-(s-1) \epsilon, \epsilon, \ldots, \epsilon, 0 \ldots 0)$ (only first $s$ entries are nonzero). Proposition 3 then follows.

\section{APPENDIX IV}

Proof of (26): Consider the problem of minimizing $E\left[\kappa_{r}^{2}\left\|\mathbf{Q}_{\mathrm{opt}}-\mathbf{Y}\right\|_{F}^{2} \mid \mathbf{Q}_{\mathrm{opt}} \in \mathcal{Q}_{k}\right]$ w.r.t. the matrix $\mathbf{Y}$. The solution to this problem can be easily shown to be $\mathbf{Y}_{\mathrm{opt}}=E\left[\kappa_{r}^{2} \mathbf{Q}_{\mathrm{opt}} \mid \mathbf{Q}_{\mathrm{opt}} \in \mathcal{Q}_{k}\right] / E\left[\kappa_{r}^{2} \mid \mathbf{Q}_{\mathrm{opt}} \in \mathcal{Q}_{k}\right]$. It can be easily shown that $\mathbf{Y}_{\text {opt }}$ is positive semidefinite and $\operatorname{Trace}\left(\mathbf{Y}_{\mathrm{opt}}\right)=P$. Thus, $\mathbf{Y}_{\mathrm{opt}} \in \mathcal{Q}$ and (26) follows.

Proof of (28): The solution to the minimization of $E\left[\kappa_{r}^{2}\|\widetilde{\mathbf{A}}-\mathbf{Y}\|_{F}^{2} \mid \widetilde{\mathbf{A}} \in \mathcal{A}_{k}\right]$ w.r.t. the matrix $\mathbf{Y}$ can be shown to be $\mathbf{Y}_{\text {opt }}=E\left[\kappa_{r}^{2} \widetilde{\mathbf{A}} \mid \widetilde{\mathbf{A}} \in \mathcal{A}_{k}\right] / E\left[\kappa_{r}^{2} \mid \widetilde{\mathbf{A}} \in \mathcal{A}_{k}\right]$. It can be deduced that $\mathbf{Y}_{\text {opt }}$ is positive definite. To show that $\mathbf{Y}_{\text {opt }} \in \mathcal{A}$, it suffices to show that $\mathbf{Q}_{Y} \in \mathcal{Q}$, where $\mathbf{Q}_{Y}=-\mathbf{Y}_{\text {opt }}+\left(\operatorname{Trace}\left(\mathbf{Y}_{\text {opt }}\right) / m\right) \mathbf{I}_{m}+(P / m) \mathbf{I}_{m}$. It can be proved that $\mathbf{Q}_{Y}=E\left(\kappa_{r}^{2} \mathbf{Q}_{\widetilde{\mathbf{A}}} \mid \widetilde{\mathbf{A}} \in \mathcal{A}_{k}\right) / E\left[\kappa_{r}^{2} \mid \widetilde{\mathbf{A}} \in \mathcal{A}_{k}\right]$, where $\mathbf{Q}_{\widetilde{A}}=-\widetilde{\mathbf{A}}+(\operatorname{Trace}(\widetilde{\mathbf{A}}) / m) \mathbf{I}_{m}+(P / m) \mathbf{I}_{m}$. Similar to Appendix IV-A, it can be argued that $\mathbf{Q}_{Y} \in \mathcal{Q}$. Hence, $\mathbf{Y} \in \mathcal{A}$ and (28) follows.

\section{REFERENCES}

[1] I. E. Telatar, "Capacity of multi-antenna Gaussian channels," Eur. Trans. Telecommun., vol. 10, no. 6, pp. 585-596, Nov.-Dec. 1999.

[2] G. J. Foschini and M. J. Gans, "On limits of wireless communications in a fading environment when using multiple antennas," Wireless Pers. Commun., no. 6, pp. 311-335, 1998.

[3] E. Biglieri, G. Caire, and G. Taricco, "Limiting performance of blockfading channels with multiple antennas," IEEE Trans. Inf. Theory, vol. 47, no. 5, pp. 1273-1289, May 2001.

[4] A. Goldsmith, S. A. Jafar, N. Jindal, and S. Vishwanath, "Capacity limits of MIMO channels," IEEE J. Sel. Areas Commun., vol. 21, no. 6, pp. 684-702, Jun. 2003.

[5] S. H. Simon and A. Moustakas, "Optimality of beamforming in multiple transmitter multiple receiver communication systems with partial channel knowledge," in Proc. DIMACS Workshop Signal Process. Wireless Commun., 2002, p. 57. 
[6] M. Medard, "The effect upon channel capacity in wireless communications of perfect and imperfect knowledge of the channel," IEEE Trans. Inf. Theory, vol. 47, no. 5, pp. 1273-1289, May 2001.

[7] E. Baccarelli, M. Baugi, and C. Pelizzoni, "On the information throughput and optimized power allocation for MIMO wireless systems with imperfect channel estimation," IEEE Trans. Signal Process., vol. 53, no. 7, pp. 2235-2346, Jul. 2005.

[8] A. Narula, M. J. Lopez, M. D. Trott, and G. W. Wornell, "Efficient use of side information in multiple-antenna data transmission over fading channels," IEEE J. Sel. Areas Commun., vol. 16, no. 8, pp. 1423-1436, Oct. 1998.

[9] K. K. Mukkavilli, A. Sabharwal, E. Erkip, and B. Aazhang, "On the beamforming with finite rate feedback in multiple antennas," IEEE Trans. Inf. Theory, vol. 49, no. 10, pp. 2562-2579, Oct. 2003.

[10] J. C. Roh and B. D. Rao, "Vector quantization techniques for multiple-antenna channel information feedback," in Proc. Int. Conf. Signal Process. Commun., Bangalore, India, Dec. 2004, pp. 402-406.

[11] _ , "MIMO spatial multiplexing systems with limited feedback," in Proc. IEEE Int. Conf. Commun., Seoul, Korea, May 2005, vol. 2, pp. 777-782.

[12] W. Santipach and M. L. Honig, "Asymptotic performance of MIMO wireless channels with limited feedback," in Proc. IEEE Mil. Commun. Conf., Boston, MA, Oct. 2003, pp. 141-146.

[13] S. Bhashyam, A. Sabharwal, and B. Aazhang, "Feedback gain in multiple antenna systems," IEEE Trans. Commun., vol. 50, no. 5, pp. 785-798, May 2002.

[14] V. Lau, Y. Liu, and T. A. Chen, "On the design of MIMO blockfading channels with feedback-link capacity constraint," IEEE Trans. Commun., vol. 52, no. 1, pp. 62-70, Jan. 2004.

[15] A. Gersho and R. M. Gray, Vector Quantization and Signal Compression. Norwell, MA: Kluwer, 1992.

[16] D. J. Love, R. W. Heath, W. Santipach, and M. L. Honig, "What is the value of limited feedback for MIMO channels," IEEE Commun. Mag., vol. 42, no. 10, pp. 54-59, Oct. 2004.

[17] J. C. Roh and B. D. Rao, "An efficient feedback method for MIMO systems with slowly time-varying channels," in Proc. IEEE Wireless Commun. Netw. Conf., Atlanta, GA, Mar. 2004, pp. 760-764.

[18] R. A. Horn and C. R. Johnson, Matrix Analysis. Cambridge, U.K.: Cambridge Univ. Press, 1990.

[19] T. M. Cover and J. A. Thomas, Elements of Information Theory. New York: Wiley, 1991.

[20] R. Bhatia, Matrix Analysis. New York: Springer Verlag, 1996.

[21] M. R. Bhavani Shankar and K. V. S. Hari, "On the variations in capacity of MIMO communication systems to channel perturbations," in Proc. IEEE ICPWC, New Delhi, India, Dec. 2002, pp. 76-80.

[22] J. H. Wilkinson, The Algebraic Eigenvalue Problem. Oxford, U.K.: Clarendon, 1965.

[23] H. Bolcskei, D. Gesbert, and A. Paulraj, "On the capacity of OFDMbased spatial multiplexing systems," IEEE Trans. Commun., vol. 50, no. 2, pp. 225-234, Feb. 2002.
[24] D. Luenberger, Linear and Non-Linear Programming. Reading, MA: Addison-Wesley, 1984.

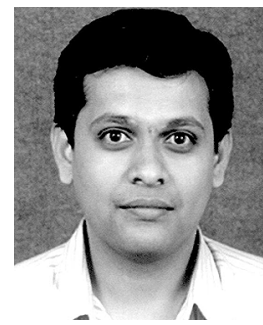

M. R. Bhavani Shankar received the B.E. degree in electronics and communication engineering from the National Institute of Technology (NIT), Trichy, India, in 1998, and the M.E. degree in signal processing in 2000 from the Indian Institute of Science (IISc), Bangalore, India, where he is currently working toward the Ph.D. degree in the Department of Electrical Communication Engineering.

From 2000 to 2001, he was with Sasken Communication Technologies, Bangalore, India, where he was involved with development of audio coders. His research interests include signal processing, wireless communications, and MIMO systems.

Mr. Shankar received the Gold Medal for Academic Excellence from IISc in 2000.

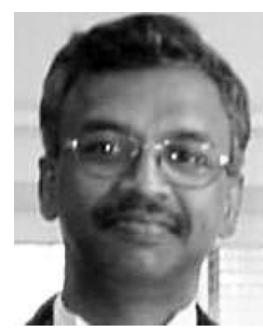

K. V. S. Hari (SM'97) received the B.E. degree from Osmania University, Hyderabad, India, in 1983, the M.Tech. degree from the Indian Institute of Technology, New Delhi, India (IIT Delhi), in 1985, and the Ph.D. degree from the University of California, San Diego, La Jolla, in 1990.

He has been a Professor with the Department of Electrical Communication Engineering, Indian Institute of Science (IISc), Bangalore, India, since February 1998. He has been Visiting Faculty at Helsinki University of Technology, Espoo, Finland, from May to July 2002; Stanford University, Stanford, CA, from September 1999 to December 2000; and the Royal Institute of Technology, Stockholm, Sweden, from July to September 1995. He has been an Assistant Professor with the Department of ECE, IISc, from February 1992 to January 1998; a Scientist at Osmania University, from December 1990 to January 1991; and a Scientist with the Defence Electronics Research Lab, Hyderabad, India, from December 1985 to July 1987. His research interests are in statistical signal processing. He has worked on space-time signal processing algorithms for direction-of-arrival estimation, acoustic signal separation using microphone arrays, and MIMO wireless communication systems. He has also worked on MIMO wireless channel measurements and modeling, and is the co-author of the IEEE 802.16 (WiMAX) standard on wireless channel models for fixed broadband wireless communication systems. He has been a consultant to various companies around the world, and is also a cofounder of a start-up company, ESQUBE Communication Solutions Pvt. Ltd, Bangalore, India. He is currently a member of the Editorial Board of EURASIP's Journal on Signal Processing. 